Correspondence: Prof. Cameron Jones

School of Chemistry

Monash University

P.O. Box 23

Melbourne, VIC, 3800

Australia

Email: cameron.jones@monash.edu

\title{
Synthesis and Attempted Reductions of Bulky 1,3,5-Triazapentadienyl Groups 2 and 13 Halide Complexes
}

\author{
Indrek Pernik, ${ }^{\mathrm{a}}$ Brant J. Maitland, ${ }^{\mathrm{a}}$ Andreas Stasch${ }^{*, \mathrm{a}, \mathrm{b}}$ and Cameron Jones*a \\ ${ }^{a}$ School of Chemistry, Monash University, P.O. Box 23, Melbourne, Victoria, 3800, Australia \\ Email: cameron.jones@monash.edu,Ph: +61-3-9902-0391,Fax:+61-3-9905-4597 \\ Web: www.monash.edu/science/research-groups/chemistry/jonesgroup \\ ${ }^{b}$ Current address: EaStCHEM School of Chemistry, University of St Andrews, North Haugh, \\ KY16 9ST, St Andrews, United Kingdom
}

Received: 


\begin{abstract}
Three extremely bulky 1,3,5-triazapentadienes, ${ }^{\mathrm{Ar}} \mathrm{NNNH}\left({ }^{\mathrm{Ar}} \mathrm{NNN}=\mathrm{N}\left\{\mathrm{C}\left(\mathrm{Bu}^{t}\right)=\mathrm{N}(\mathrm{Ar})\right\}_{2} ; \mathrm{Ar}=\right.$ Mes (mesityl), Dep (2,6-diethylphenyl) or Dip (2,6-diisopropylphenyl)) have been prepared and structurally characterized. These are readily deprotonated, yielding a series of lithium and potassium triazapentadienyl complexes, one of which, $\left({ }^{\mathrm{Dip}} \mathrm{NNN}\right) \mathrm{Li}$, has been structurally characterized. Similarly, three monomeric triazapentadienyl magnesium iodide complexes, $\left({ }^{\mathrm{Ar}} \mathrm{NNN}\right) \mathrm{MgI}\left(\mathrm{OEt}_{2}\right)$, and a dimeric calcium counterpart, $\left\{\left({ }^{\mathrm{Mes}} \mathrm{NNN}\right) \mathrm{Ca}(\mathrm{THF})(\mu-\mathrm{I})\right\}_{2}$, have been prepared. Attempts to reduce the former gave homoleptic bis(triazapentadienyl) magnesium complexes, $\left({ }^{\mathrm{Ar}} \mathrm{NNN}\right)_{2} \mathrm{Mg}(\mathrm{Ar}=\mathrm{Mes}$ or Dep $)$ as the main products. One reaction also gave a very low yield of the magnesium(I) dimer, $\left\{\left({ }^{\text {Dep }} \mathrm{NNN}\right) \mathrm{Mg}-\right\}_{2}$, which was structurally characterized. In related chemistry, two triazapentadienyl boron difluoride compounds, $\left({ }^{\mathrm{Ar} N N N}\right) \mathrm{BF}_{2}(\mathrm{Ar}=\mathrm{Mes}$ or Dep), have been synthesized, and unsuccessful attempts made to reduce these to boron(I) heterocycles. For sake of comparison, attempts have been made to prepare a series of related amino-substituted $\beta$-diketiminato group 13 element(I) heterocycles. While these were also not successful, several group 13 element(III) halide complexes incorporating this ligand class, and related amino-substituted $\beta$-diketiminates, have been characterized.
\end{abstract}

Key words: Triazapentadienyl, Steric bulk, $\beta$-Diketiminate, Group 2, Group 13, Magnesium(I) 


\section{Introduction}

The fundamental and applied chemistry of compounds containing main group elements in very low oxidation states and/or with very low coordination numbers has rapidly expanded in recent decades. ${ }^{1}$ This has been allowed by the development of numerous sterically bulky ligands, which kinetically protect the coordinated main group element from undergoing disproportionation processes. One of the most successful ligand class in this respect are bulky aryl substituted, bidentate $\beta$-diketiminates (Nacnacs), A (Figure 1), which also provide thermodynamic stability to the coordinated main group center through the chelate effect. Numerous examples of such ligands are known, the steric profiles of which are relatively simple to tailor through alteration of the size of either the $N$-aryl or backbone $C$-substituents. ${ }^{2}$ Tailoring the electronic properties of $\beta$-diketiminates is not as commonly carried out, though it is a strategy that is investigated. One recent example involved replacement of the Nacnac backbone organyl substituent with an amino group, to yield modified $\beta$-diketiminato ligands, $\mathrm{B}$, which are highly electron rich, by virtue of them possessing resonance structures with two negatively charged $N$ donor centers. The ligands were subsequently utilized in the stabilization of several low oxidation main group complexes. ${ }^{3}$

Another electron rich ligand class, which is closely related to $\beta$-diketiminates, are the $N, N^{\prime}$-chelating 1,3,5-triazapentadienyls, C. Although a number of these have been developed and applied in coordination chemistry, ${ }^{4}$ we are not aware of any instances where they are incorporated into unusually low oxidation state $N, N^{\prime}$-chelated main group complexes. Here we describe the syntheses of a series of extremely bulky 1,3,5-triazapentadienes and their use in the preparation of a variety of 1,3,5-triazapentadienyl groups 2 and 13 metal halide complexes. 
Although the reduction of these species to give low oxidation state complexes was largely unsuccessful, one low-yielding triazapentadienyl coordinated magnesium(I) dimer has been forthcoming.

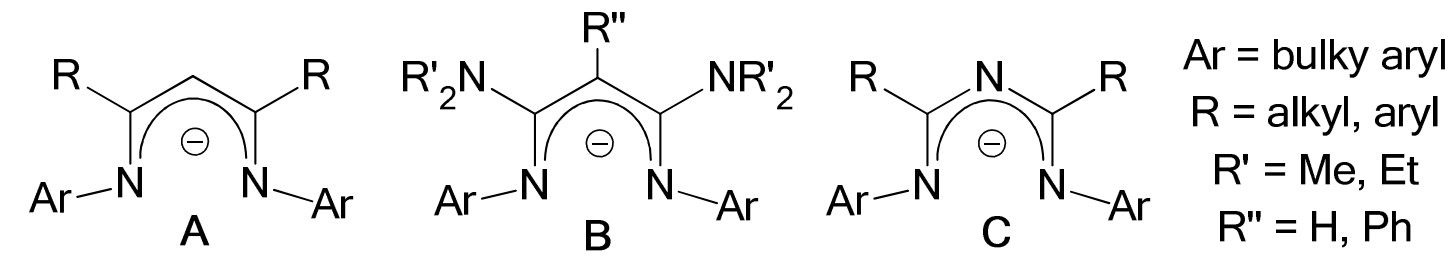

Fig. 1. Generic representations of bulky $\beta$-diketiminates (A), amino-substituted $\beta$-diketiminates

(B) and 1,3,5-triazapentadienyls (C)

\section{Experimental Section}

General methods. All manipulations were carried out using standard Schlenk and glove box techniques under an atmosphere of high purity dinitrogen. Hexane and toluene were distilled over potassium, whilst diethyl ether was distilled over $\mathrm{Na} / \mathrm{K}$ alloy (1:1). Dichloromethane was distilled over $\mathrm{CaH}_{2}$. ${ }^{1} \mathrm{H}$ and ${ }^{13} \mathrm{C}\left\{{ }^{1} \mathrm{H}\right\}$ NMR spectra were recorded on Bruker AvanceIII 400 or Varian Inova 500 spectrometers and were referenced to the resonances of the solvent used. ${ }^{11} \mathrm{~B},{ }^{19} \mathrm{~F}$ and ${ }^{7}$ Li NMR spectra were recorded on a Bruker AvanceIII 400 spectrometer and were referenced to external $\mathrm{BF}_{3}\left(\mathrm{OEt}_{2}\right), \mathrm{CFCl}_{3}$ and $1 \mathrm{M} \mathrm{LiCl}$ in $\mathrm{D}_{2} \mathrm{O}$ respectively. Mass spectra were collected using an Agilent Technologies 5975D inert MSD with a solid state probe. FTIR spectra were recorded using a Perkin-Elmer RX1 spectrometer as Nujol mulls between $\mathrm{NaCl}$ plates, or on solid samples protected from the atmosphere with a film of Nujol using an Agilent Cary 630 attenuated total reflectance (ATR) spectrometer. Microanalyses were carried out by the Science Centre, London Metropolitan University. Melting points were determined in sealed glass capillaries under dinitrogen and are uncorrected. The compounds $\mathrm{Al}^{\mathrm{I}} \mathrm{Cl}$ and $\mathrm{Ga}^{\mathrm{I}} \mathrm{Cl}$ (both as solutions in 
toluene/THF $),{ }^{5}\left[\mathrm{PhC}\left\{\mathrm{C}\left(\mathrm{NEt}_{2}\right) \mathrm{N}(\mathrm{Mes})\right\}_{2}\right] \mathrm{Li}^{3}$ and $\left[\mathrm{HC}\left\{\mathrm{C}\left(\mathrm{NEt}_{2}\right) \mathrm{N}(\mathrm{Mes})\right\}_{2}\right] \mathrm{Li}^{3}$ were prepared by literature procedures. Imidoyl chloride and amidine precursors to ${ }^{\mathrm{Ar}} \mathrm{NNNH}$ were prepared by variations of literature procedures. ${ }^{6-8}$ All other reagents were used as received.

\begin{abstract}
${ }^{\text {Mes NNNH }}$
$\operatorname{MesNC}(\mathrm{Cl}) \mathrm{Bu}^{t}(10.7 \mathrm{~g}, 44.8 \mathrm{mmol})$ and $\mathrm{MesNC}\left(\mathrm{NH}_{2}\right) \mathrm{Bu}^{t}(9.8 \mathrm{~g}, 44.8 \mathrm{mmol})$ were heated in a melt at $200{ }^{\circ} \mathrm{C}$ for three hours under a nitrogen atmosphere. After being cooled to room temperature, the resultant solid was dissolved in dichloromethane $(70 \mathrm{~mL})$, the extract washed with aqueous potassium carbonate solution $(1 \mathrm{M}, 20 \mathrm{~mL})$, dried $\left(\mathrm{MgSO}_{4}\right)$, and then volatiles removed in vacuo to give an off-white solid. The crude product was recrystallized from boiling ethyl acetate to give the product as a colourless solid (14.8 g, $79 \%$ \%. M.p. $173-175{ }^{\circ} \mathrm{C} ;{ }^{1} \mathrm{H}$ NMR $\left(400 \mathrm{MHz}, 298 \mathrm{~K}, \mathrm{C}_{6} \mathrm{D}_{6}\right): \delta 0.89\left(\mathrm{v} . \mathrm{br}, 9 \mathrm{H}, \mathrm{C}\left(\mathrm{CH}_{3}\right)_{3}\right), 1.46\left(\mathrm{~s}, 9 \mathrm{H}, \mathrm{C}\left(\mathrm{CH}_{3}\right)_{3}\right), 1.96$ (v. br, 12H, Ar- $\left.\mathrm{CH}_{3}\right), 2.12\left(\mathrm{~s}, 3 \mathrm{H}, \mathrm{Ar}-\mathrm{CH}_{3}\right), 2.26$ (s, 3H, Ar-CH$), 4.75$ (br, 1H, NH), 6.75 (s, 2H, ArH), 6.81 $(\mathrm{s}, 2 \mathrm{H}, \mathrm{Ar} H) ;{ }^{13} \mathrm{C}\left\{{ }^{1} \mathrm{H}\right\}\left(101 \mathrm{MHz}, 298 \mathrm{~K}, \mathrm{CDCl}_{3}\right): \delta 18.5\left(\mathrm{Ar}-\mathrm{CH}_{3}\right), 19.0\left(\mathrm{Ar}-\mathrm{CH}_{3}\right), 21.0(\mathrm{Ar}-$ $\left.\mathrm{CH}_{3}\right), 29.0\left(\mathrm{CCH}_{3}\right), 29.6\left(\mathrm{CCH}_{3}\right), 39.1\left(\mathrm{CCH}_{3}\right), 39.7\left(\mathrm{CCH}_{3}\right), 128.6,128.7,128.8,128.9,131.5$, 136.2 (Ar-C), $136.7(\mathrm{NCN}), 137.0(\mathrm{NCN}) ; \mathrm{IR} v / \mathrm{cm}^{-1}$ (Nujol): $3416(\mathrm{~N}-\mathrm{H}$ str, m), 1670s, 1589s, 1478m, 1103s, 962m, 848m, 787m, 739w, 675w; MS/ESI m/z (\%): $419.5\left(\mathrm{M}^{+}, 100\right)$; acc. mass MS/ESI calc. for $\mathrm{C}_{28} \mathrm{H}_{42} \mathrm{~N}_{3}{ }^{+}: 420.3379$, found: 420.3373 .
\end{abstract}

\title{
${ }^{\text {Dep NNNH }}$
}

Prepared using a similar procedure for the preparation of ${ }^{\mathrm{Mes}} \mathrm{NNNH}$, but using $\mathrm{DepNC}(\mathrm{Cl}) \mathrm{Bu}^{t}$ (8.60 g, $34.2 \mathrm{mmol})$ and $\mathrm{DepNC}\left(\mathrm{NH}_{2}\right) \mathrm{Bu}^{t}(7.94 \mathrm{~g}, 34.2 \mathrm{mmol})$. The title compound was obtained as a colorless crystalline solid (13.0 g, 84\%). M.p. 96-98 ${ }^{\circ} \mathrm{C} ;{ }^{1} \mathrm{H}$ NMR $(300 \mathrm{MHz}, 298 \mathrm{~K}$, $\mathrm{CDCl}_{3}$ ): $\delta 1.15$ (virt. t, ${ }^{3} J=7.6 \mathrm{~Hz}, 12 \mathrm{H}, \mathrm{CH}_{2} \mathrm{CH}_{3}$ ), 1.25 (s, 9H, $\left.\mathrm{C}\left(\mathrm{CH}_{3}\right)_{3}\right), 1.29$ (br, 9H, 
$\left.\mathrm{C}\left(\mathrm{CH}_{3}\right)_{3}\right), 2.44$ (br. m, 8H, $\mathrm{CH}_{2} \mathrm{CH}_{3}$ ), 6.94 (br, 2H, $\mathrm{ArH}$ ), 7.04 (br., 4H, $\mathrm{ArH}$ ), $\mathrm{NH}$ resonance not observed; ${ }^{13} \mathrm{C}\left\{{ }^{1} \mathrm{H}\right\}$ NMR $\left(101 \mathrm{MHz}, 348 \mathrm{~K}, \mathrm{CDCl}_{3}\right): \delta 14.0\left(\mathrm{CH}_{2} \mathrm{CH}_{3}\right), 14.5\left(\mathrm{CH}_{2} \mathrm{CH}_{3}\right), 24.5$

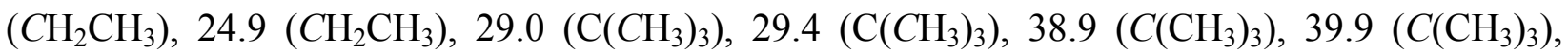
121.7, 225.9, 127.5, 133.8, 142.6, 146.3, $154.8(\mathrm{NCN}) ; \mathrm{IR} v / \mathrm{cm}^{-1}$ (Nujol): 3424 (N-H str, m), $1671 \mathrm{~s}, 1597 \mathrm{~s}, 1580(\mathrm{~s}), 1510(\mathrm{~s}), 1216 \mathrm{~m}, 1097 \mathrm{~s}, 954 \mathrm{~s}, 800 \mathrm{~s}, 753 \mathrm{~s}, 696 \mathrm{~m} ; \mathrm{MS} / \mathrm{ESI} \mathrm{m} / z(\%): 447.7$ $\left(\mathrm{M}^{+}, 100\right)$; acc. mass MS/ESI calc. for $\mathrm{C}_{30} \mathrm{H}_{46} \mathrm{~N}_{3}{ }^{+}:$448.3692, found: 448.3685 .

\begin{abstract}
${ }^{\text {Dip }}$ NNNH
Prepared using a similar procedure for the preparation of ${ }^{\mathrm{Mes}} \mathrm{NNNH}$, but using $\operatorname{DipNC}(\mathrm{Cl}) \mathrm{Bu}^{t}$ (2.47 $\mathrm{g}, 9.5 \mathrm{mmol})$ and $\operatorname{DipNC}\left(\mathrm{NH}_{2}\right) \mathrm{Bu}^{t}(2.65 \mathrm{~g}, 9.5 \mathrm{mmol})$. The title compound was obtained, with poor reproducibility, as an impure white solid after work-up. On one occassion a small quantity of pure crystals were obtained after recrystallization from ethanol. M.p. $225-227{ }^{\circ}$ C; MS/ESI m/z (\%): $503.6\left(\mathrm{MH}^{+}, 100\right)$; anal. calc. for $\mathrm{C}_{34} \mathrm{H}_{53} \mathrm{~N}_{3}$ : C 81.06\%, $\mathrm{H} 10.60 \%, \mathrm{~N} \mathrm{8.34 \%}$; found: C $80.95 \%$, H 10.81\%, 8.31\%. N.B. No other spectroscopic data could be reliably assigned due to the poor reproducibility of the compound synthesis, and the fact that when formed it was always contaminated with significant amounts of unidentified products. The impure product mix containing ${ }^{\text {Dip }} \mathrm{NNNH}$ was used in subsequent syntheses.
\end{abstract}

\title{
$\left({ }^{\mathrm{Mes}} \mathrm{NNN}\right) \mathrm{Li} \cdot \mathrm{OEt}_{2}$ (1a)
}

To a solution of ${ }^{\mathrm{Mes}} \mathrm{NNNH}(1.0 \mathrm{~g}, 2.38 \mathrm{mmol})$ in diethyl ether $(20 \mathrm{~mL})$ at $-80{ }^{\circ} \mathrm{C}$ was added a solution of $\mathrm{Bu}^{n} \mathrm{Li}$ in hexane $(1.58 \mathrm{~mL}, 1.65 \mathrm{M}, 2.53 \mathrm{mmol})$. The resultant solution was warmed to room temperature and left to stir overnight. The reaction mixture was then concentrated in vacuo, leading to the formation of $\mathbf{1 a}$ as a white precipitate. This was collected by filtration. A second crop of 1a, as a colourless crystalline solid, was obtained by cooling the mother liquor to $2{ }^{\circ} \mathrm{C}$ 
overnight (0.65 g, 55\%). M.p. $>260{ }^{\circ} \mathrm{C} ;{ }^{1} \mathrm{H}$ NMR (400 MHz, $\left.298 \mathrm{~K}, \mathrm{C}_{6} \mathrm{D}_{6}\right): \delta 0.52\left(\mathrm{t},{ }^{3} J=6.7\right.$ $\left.\mathrm{Hz}, 6 \mathrm{H}, \mathrm{CH}_{3} \mathrm{CH}_{2} \mathrm{O}\right), 1.43\left(\mathrm{~s}, 18 \mathrm{H}, \mathrm{C}\left(\mathrm{CH}_{3}\right)_{3}\right), 2.19$ (s, 12H, Ar- $\left.\mathrm{CH}_{3}\right), 2.21\left(\mathrm{~s}, 6 \mathrm{H}, \mathrm{Ar}-\mathrm{CH}_{3}\right), 2.61$ $\left(\mathrm{q},{ }^{3} J=6.7 \mathrm{~Hz}, 4 \mathrm{H}, \mathrm{OCH} H_{2}\right), 6.61-6.74(\mathrm{~m}, 4 \mathrm{H}, \mathrm{Ar}-H) ;{ }^{13} \mathrm{C}\left\{{ }^{1} \mathrm{H}\right\} \mathrm{NMR}\left(100 \mathrm{MHz}, 298 \mathrm{~K}, \mathrm{C}_{6} \mathrm{D}_{6}\right): \delta$ $13.7\left(\mathrm{OCH}_{2} \mathrm{CH}_{3}\right), 19.1\left(\mathrm{Ar}-\mathrm{CH}_{3}\right), 20.6\left(\mathrm{Ar}-\mathrm{CH}_{3}\right), 31.0\left(\mathrm{C}\left(\mathrm{CH}_{3}\right)_{3}\right), 44.3\left(\mathrm{C}_{\left.\left(\mathrm{CH}_{3}\right)_{3}\right),}, 65.1\left(\mathrm{OCH}_{2}\right)\right.$, 128.2, 124.4, 128.5, $148.4(\mathrm{Ar}-\mathrm{C}), 170.1(\mathrm{NCN}) ;{ }^{7} \mathrm{Li} \mathrm{NMR}\left(154 \mathrm{MHz}, 298 \mathrm{~K}, \mathrm{C}_{6} \mathrm{D}_{6}\right): \delta 1.25 ; \mathrm{IR}$ $\mathrm{v} / \mathrm{cm}^{-1}$ (ATR): 1064m, 1022m, 1006m, 950w, 931w, 909m, 878m, 858s, 836m, 791w, 758m, 733s; MS/EI $m / z(\%): 419.3\left({ }^{\mathrm{Mes}} \mathrm{NNN}^{+}, 20\right), 362.4\left({ }^{\mathrm{Mes}} \mathrm{NNN}^{+}-\mathrm{Bu}^{t}, 100\right), 285.3\left({ }^{\mathrm{Mes}} \mathrm{NNN}^{+}-\mathrm{NMes}\right.$ 29); anal. calc. for $\mathrm{C}_{32} \mathrm{H}_{51} \mathrm{LiN}_{3} \mathrm{O}$ : C 76.76\%, H 10.27\%, N 8.39\%; found: C 77.83\%, H 9.62\%, N $9.72 \%$.

\section{$\left({ }^{\mathrm{Dep}} \mathrm{NNN}\right) \mathrm{Li} \cdot \mathrm{OEt}_{2}(\mathbf{1 b})$}

Prepared using a similar procedure for the preparation of 1a, but using ${ }^{\text {Dep }} \mathrm{NNNH}(4.0 \mathrm{~g}, 8.93$ $\mathrm{mmol})$ and $\mathrm{Bu}^{n} \mathrm{Li}$ in hexane $(6.2 \mathrm{~mL}, 1.6 \mathrm{M}, 9.82 \mathrm{mmol})$. The title compound was obtained as a pale yellow crystalline solid after work-up (3.80 g, 81\%). M.p. $>260{ }^{\circ} \mathrm{C} ;{ }^{1} \mathrm{H} \mathrm{NMR}(300 \mathrm{MHz}$, $\left.298 \mathrm{~K}, \mathrm{C}_{6} \mathrm{D}_{6}\right): \delta 0.44\left(\mathrm{t},{ }^{3} \mathrm{~J}=7.0 \mathrm{~Hz}, 6 \mathrm{H}, \mathrm{OCH}_{2} \mathrm{CH}_{3}\right), 1.20\left(\mathrm{t},{ }^{3} \mathrm{~J}=7.6 \mathrm{~Hz}, 12 \mathrm{H}, \mathrm{CH}_{2} \mathrm{CH}_{3}\right), 1.42(\mathrm{~s}$, $\left.18 \mathrm{H}, \mathrm{C}\left(\mathrm{CH}_{3}\right)_{3}\right), 2.54\left(\mathrm{q},{ }^{3} \mathrm{~J}=7.0 \mathrm{~Hz}, 4 \mathrm{H}, \mathrm{OCH}_{2}\right), 2.66\left(\mathrm{q},{ }^{3} \mathrm{~J}=7.6 \mathrm{~Hz}, 8 \mathrm{H}, \mathrm{CH}_{2} \mathrm{CH}_{3}\right), 6.95-7.04$ $(\mathrm{m}, 6 \mathrm{H}, \mathrm{Ar}-H) ;{ }^{13} \mathrm{C}\left\{{ }^{1} \mathrm{H}\right\} \mathrm{NMR}\left(75 \mathrm{MHz}, 298 \mathrm{~K}, \mathrm{C}_{6} \mathrm{D}_{6}\right): \delta 13.5\left(\mathrm{OCH}_{2} \mathrm{CH}_{3}\right), 13.8\left(\mathrm{CH}_{2} \mathrm{CH}_{3}\right), 25.0$ $\left(\mathrm{CH}_{2} \mathrm{CH}_{3}\right), 31.6\left(\mathrm{C}\left(\mathrm{CH}_{3}\right)_{3}\right), 44.9\left(\mathrm{C}_{\left.\left(\mathrm{CH}_{3}\right)_{3}\right),} 64.5\left(\mathrm{OCH}_{2}\right), 121.8,125.0,134.2,150.2\right.$ (Ar- $\left.\mathrm{C}\right)$, $169.9(\mathrm{NCN}) ;{ }^{7} \mathrm{Li}$ NMR (154 MHz, $\left.298 \mathrm{~K}, \mathrm{C}_{6} \mathrm{D}_{6}\right): \delta 1.09 ; \mathrm{IR} v / \mathrm{cm}^{-1}$ (ATR): 1192w, 1138w, 1115m, 1099m, 967m, 861w, 808s, 753s, 712w; MS/EI m/z (\%): $453.3\left(\mathrm{M}^{+}-\mathrm{OEt}_{2}, 0.1\right), 447.4$ $\left({ }^{\mathrm{Dep}} \mathrm{NNNH}^{+}, 17\right), 418.4\left({ }^{\mathrm{Dep}} \mathrm{NNN}^{+}-\mathrm{Et}, 9\right), 390.4\left({ }^{\mathrm{Dep}} \mathrm{NNN}^{+}-\mathrm{Bu}^{t}, 100\right)$; anal. calc. for $\mathrm{C}_{30} \mathrm{H}_{45} \mathrm{LiN}_{3}$ : C 79.25\%, H 9.98\%, N 9.24\%; found: C 79.27\%, H 9.02\%, N 9.72\%, N.B. diethyl ether of coordination was readily lost during the drying process. 


\section{$\left({ }^{\mathrm{Dip}} \mathrm{NNN}\right) \mathbf{L i}(\mathbf{1 c})$}

Prepared using a similar procedure for the preparation of 1a, but using impure ${ }^{\text {Dip }} \mathrm{NNNH}(0.9 \mathrm{~g})$ and $\mathrm{Bu}^{n} \mathrm{Li}$ in hexane $(1.40 \mathrm{~mL}, 1.6 \mathrm{M}, 2.20 \mathrm{mmol})$. The title compound was obtained as a pure colorless crystalline solid after recrystallization from hexane $(0.35 \mathrm{~g}, \mathrm{ca} .37 \%)$. M.p. $209-212{ }^{\circ} \mathrm{C}$;

${ }^{1} \mathrm{H}$ NMR (400 MHz, $\left.303 \mathrm{~K}, \mathrm{C}_{6} \mathrm{D}_{6}\right): \delta 0.93\left(\mathrm{~d},{ }^{3} \mathrm{~J}=6.8 \mathrm{~Hz}, 12 \mathrm{H}, \mathrm{CH}\left(\mathrm{CH}_{3}\right)_{2}\right), 1.31\left(\mathrm{~d},{ }^{3} \mathrm{~J}=6.8 \mathrm{~Hz}\right.$, 12H, $\left.\mathrm{CH}\left(\mathrm{CH}_{3}\right)_{2}\right), 1.43\left(\mathrm{~s}, 18 \mathrm{H}, \mathrm{C}\left(\mathrm{CH}_{3}\right)_{3}\right), 3.29$ (sept, $\left.{ }^{3} \mathrm{~J}=6.8 \mathrm{~Hz}, 4 \mathrm{H}, \mathrm{CH}\left(\mathrm{CH}_{3}\right)_{2}\right), 7.04(\mathrm{~m}, 6 \mathrm{H}$, $\mathrm{ArH}) ;{ }^{13} \mathrm{C}\left\{{ }^{1} \mathrm{H}\right\}$ NMR $\left(100 \mathrm{MHz}, 303 \mathrm{~K}, \mathrm{C}_{6} \mathrm{D}_{6}\right): \delta 21.7\left(\mathrm{C}\left(\mathrm{CH}_{3}\right)_{3}\right), 24.0\left(\mathrm{CH}\left(\mathrm{CH}_{3}\right)_{2}\right), 26.7$

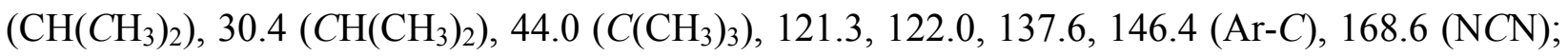
${ }^{7} \mathrm{Li}$ NMR (154 MHz, $303 \mathrm{~K}, \mathrm{C}_{6} \mathrm{D}_{6}$ ): $\delta 1.35$; IR v/cm $\mathrm{cm}^{-1}$ (ATR): 1617w, 1391w, 1248m, 1035m, 895s, 832s, 752s, 683m; MS/EI m/z (\%): $504.4\left({ }^{\mathrm{Dip}} \mathrm{NNNH}^{+}, 100 \%\right)$; anal. calc. for $\mathrm{C}_{34} \mathrm{H}_{52} \mathrm{~N}_{3} \mathrm{Li}: \mathrm{C}$ 80.11\%, H 10.28\%, N 8.24\%; found: C 79.81\%, H 10.12\%, N 8.27\%.

\section{$\left({ }^{\text {Mes }}\right.$ NNN)K (2a)}

A Schlenk flask was charged with ${ }^{\mathrm{Mes}} \mathrm{NNNH}(1.50 \mathrm{~g}, 3.57 \mathrm{mmol})$, and $\mathrm{K}\left[\mathrm{N}\left(\mathrm{SiMe}_{3}\right)_{2}\right](0.71 \mathrm{~g}$, $3.57 \mathrm{mmol}$ ). The flask was cooled to $-20{ }^{\circ} \mathrm{C}$ and toluene (also pre-cooled to $-20^{\circ} \mathrm{C}$ ) was added. This resulted in a yellow solution which was allowed to stir overnight at room temperature. Volatiles were then removed in vacuo and the resultant oil was washed with hexane. After heating the oil for $70{ }^{\circ} \mathrm{C}$ for $1 \mathrm{hr}$ in vacuo, 2a was left as a white solid (1.10 g, 66 \%). M.p. 162$167{ }^{\circ} \mathrm{C} ;{ }^{1} \mathrm{H}$ NMR $\left(300 \mathrm{MHz}, 298 \mathrm{~K}, \mathrm{C}_{6} \mathrm{D}_{6}\right): \delta 1.12\left(\mathrm{~s}, 18 \mathrm{H}, \mathrm{C}\left(\mathrm{CH}_{3}\right)_{3}\right), 1.93\left(\mathrm{~s}, 12 \mathrm{H}, \mathrm{Ar}-\mathrm{CH}_{3}\right)$, $2.24\left(\mathrm{~s}, 6 \mathrm{H}, \mathrm{Ar}-\mathrm{CH}_{3}\right), 6.80(\mathrm{~s}, 4 \mathrm{H}, \mathrm{Ar} H) ;{ }^{13} \mathrm{C}\left\{{ }^{1} \mathrm{H}\right\}$ NMR $\left(100 \mathrm{MHz}, 303 \mathrm{~K}, \mathrm{C}_{6} \mathrm{D}_{6}\right): \delta 19.4(\mathrm{Ar}-$ $\left.\mathrm{CH}_{3}\right), 21.0\left(\mathrm{Ar}-\mathrm{CH}_{3}\right), 31.0\left(\mathrm{C}\left(\mathrm{CH}_{3}\right)_{3}\right), 40.5\left(C\left(\mathrm{CH}_{3}\right)_{3}\right), 129.2,129.4,130.0,149.0,162.6(\mathrm{NCN})$; IR $v / \mathrm{cm}^{-1}$ (ATR): 1590m, 1354m, 1296w, 1238m, 1205w, 1154w, 1106m, 1030w, 1007w, 941m, 923m, 851m; MS/EI m/z (\%): $419.4\left({ }^{\mathrm{Mes}} \mathrm{NNN}^{+}, 100\right)$. A reproducible microanalysis could not be obtained due to the highly air and moisture sensitive nature of the compound. 


\section{$\left({ }^{\mathrm{Dep}} \mathrm{NNN}\right) \mathrm{K}$ (2b)}

Prepared using a similar procedure for the preparation of $\mathbf{2 a}$, but using ${ }^{\text {Dep }}{ }^{2 N N H}(1.12$ g, 2.73 mmol) and $\mathrm{K}\left[\mathrm{N}\left(\mathrm{SiMe}_{3}\right)_{2}\right](0.50 \mathrm{~g}, 2.73 \mathrm{mmol})$. The title compound was obtained as a white solid after work-up (0.73 g, 60\%). M.p. $>260{ }^{\circ} \mathrm{C} ;{ }^{1} \mathrm{H}$ NMR $\left(400 \mathrm{MHz}, 298 \mathrm{~K}, \mathrm{C}_{6} \mathrm{D}_{6}\right): \delta 1.10$ (br. t, ${ }^{3} J=$ $\left.7.2 \mathrm{~Hz}, 12 \mathrm{H}, \mathrm{CH}_{2} \mathrm{CH}_{3}\right), 1.24\left(\mathrm{~s}, 18 \mathrm{H}, \mathrm{C}\left(\mathrm{CH}_{3}\right)_{3}\right), 2.41\left(\mathrm{br}, 8 \mathrm{H}, \mathrm{CH}_{2} \mathrm{CH}_{3}\right), 6.85\left(\mathrm{t},{ }^{3} \mathrm{~J}=7.4 \mathrm{~Hz}, 2 \mathrm{H}\right.$, $\mathrm{ArH}), 7.00\left(\mathrm{~d},{ }^{3} \mathrm{~J}=7.4 \mathrm{~Hz}, 4 \mathrm{H}, \mathrm{Ar} H\right)$; a meaningful ${ }^{13} \mathrm{C}$ NMR spectrum could not be obtained due to the low solubility of the compound in $\mathrm{C}_{6} \mathrm{D}_{6} ; \mathrm{IR} v / \mathrm{cm}^{-1}$ (ATR): $1580 \mathrm{~m}, 1522 \mathrm{w}, 1097 \mathrm{~m}, 1026 \mathrm{w}$, 1003w, 954m, 936m, 798m, 745s, 692m; MS/EI m/z (\%): $485.3\left(\mathrm{M}^{+}, 2\right), 447.4\left({ }^{\mathrm{Dep}} \mathrm{NNNH}^{+}, 14\right)$, $390.3\left(\mathrm{M}^{+}-\mathrm{Bu}^{t}, 100\right), 299.3\left(\mathrm{M}^{+}\right.$-NDep, 13); A reproducible microanalysis could not be obtained due to the highly air and moisture sensitive nature of the compound.

\section{$\left({ }^{\mathrm{Mes}} \mathrm{NNN}\right) \mathrm{MgI}\left(\mathrm{OEt}_{2}\right)(3 \mathrm{a})$}

MeMgI (1.8 mL, 3.0 M, $5.4 \mathrm{mmol})$ in diethyl ether was added to a solution of ${ }^{\mathrm{Mes}} \mathrm{NNNH}(2.0 \mathrm{~g}$, $4.76 \mathrm{mmol})$ in diethyl ether $(20 \mathrm{~mL})$ at $-60{ }^{\circ} \mathrm{C}$. The reaction mixture was warmed to room temperature, which led to the formation of white precipitate. The suspension was left standing in a fridge overnight followed by a filtration of the preciptate at $0{ }^{\circ} \mathrm{C}$. The obtained white solid was dried in vacuo $\left(2.4\right.$ g, 78 \%). M.p. $=253-256{ }^{\circ} \mathrm{C} ;{ }^{1} \mathrm{H}$ NMR $\left(300 \mathrm{MHz}, 298 \mathrm{~K}, \mathrm{C}_{6} \mathrm{D}_{6}\right): \delta 0.55$ (br, $\left.\left.6 \mathrm{H}, \mathrm{OCH}_{2} \mathrm{CH}_{3}\right), 1.28\left(\mathrm{~s}, 18 \mathrm{H}, \mathrm{C}\left(\mathrm{CH}_{3}\right)_{3}\right)\right), 2.13$ (s, 6H, Ar- $\left.\mathrm{CH}_{3}\right), 2.29$ (br, 6H, Ar- $\left.\mathrm{CH}_{3}\right), 2.58$ (br, $\left.6 \mathrm{H}, \mathrm{Ar}-\mathrm{CH}_{3}\right), 3.23\left(\mathrm{q},{ }^{3} \mathrm{~J}=7 \mathrm{~Hz}, 4 \mathrm{H}, \mathrm{OCH}_{2} \mathrm{CH}_{3}\right), 6.72(\mathrm{~s}, 4 \mathrm{H}, \mathrm{Mes}-H) ;{ }^{13} \mathrm{C}\left\{{ }^{1} \mathrm{H}\right\} \mathrm{NMR}(100 \mathrm{MHz}$, $\left.303 \mathrm{~K}, \mathrm{C}_{6} \mathrm{D}_{6}\right): \delta 13.2\left(\mathrm{OCH}_{2} \mathrm{CH}_{3}\right), 20.1\left(\mathrm{Ar}-\mathrm{CH}_{3}\right), 20.9\left(\mathrm{Ar}-\mathrm{CH}_{3}\right), 22.2\left(\mathrm{Ar}-\mathrm{CH}_{3}\right), 30.8\left(\mathrm{C}\left(\mathrm{CH}_{3}\right)_{3}\right)$,

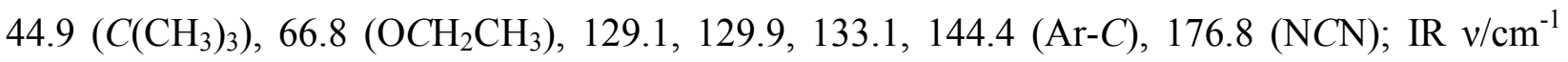
(ATR): 1524m, 1476m, 1189s, 1140s, 1090m, 1033s, 956w, 933w, 904m, 882s, 860s, 840m, 787s; MS/EI $m / z(\%): 443.3\left({ }^{\mathrm{Mes}} \mathrm{NNNMgH}^{+},<1\right), 419.4\left({ }^{\mathrm{Mes}} \mathrm{NNNH}^{+}, 11\right), 362.3\left({ }^{\mathrm{Mes}} \mathrm{NNNH}^{+}-\mathrm{Bu} t\right.$, 
100). N.B. A reproducible microanalysis could not be obtained for the compound as it consistently co-crystallised with small amounts (ca. $5 \%$ ) of protonated ligand, ${ }^{\mathrm{Mes}} \mathrm{NNNH}$, which proved inseparable, even after several attempted fractional crystallizations.

\section{$\left({ }^{\text {Dep }} \mathrm{NNN}\right) \mathrm{MgI}\left(\mathrm{OEt}_{2}\right)(3 \mathrm{~b})$}

Prepared using a similar procedure for the preparation of 3a, but using ${ }^{\text {Dep }} \mathrm{NNNH}(2.0 \mathrm{~g}, 4.47$ mmol) and MeMgI (1.64 mL, 3.0 M, $4.92 \mathrm{mmol})$ in diethyl ether. The title compound was obtained as a white solid after work-up (2.60 g, 87\%). M.p. $>260{ }^{\circ} \mathrm{C} ;{ }^{1} \mathrm{H}$ NMR $(400 \mathrm{MHz}, 298$ $\left.\mathrm{K}, \mathrm{C}_{6} \mathrm{D}_{6}\right): \delta 0.34\left(\mathrm{br}, 6 \mathrm{H}, \mathrm{OCH}_{2} \mathrm{CH}_{3}\right), 1.09\left(\mathrm{~s}, 18 \mathrm{H}, \mathrm{C}\left(\mathrm{CH}_{3}\right)_{3}\right), 1.13$ (br m, 12H, $\left.\mathrm{CH}_{2} \mathrm{CH}_{3}\right), 2.50$ (br, 4H, $\mathrm{CH}_{2} \mathrm{CH}_{3}$ ), 2.75 (br, $2 \mathrm{H}, \mathrm{CH}_{2} \mathrm{CH}_{3}$ ), 3.03 (q, ${ }^{3} \mathrm{~J}=7.0 \mathrm{~Hz}, 4 \mathrm{H}, \mathrm{OCH}_{2} \mathrm{CH}_{3}$ ), 3.13 (br, 2H, $\left.\mathrm{CCH}_{2} \mathrm{CH}_{3}\right), 6.90$ (br, 6H, ArH); ${ }^{13} \mathrm{C}\left\{{ }^{1} \mathrm{H}\right\} \mathrm{NMR}\left(100 \mathrm{MHz}, 303 \mathrm{~K}, \mathrm{C}_{6} \mathrm{D}_{6}\right): \delta 13.2\left(\mathrm{CH}_{2} \mathrm{CH}_{3}\right), 13.3$

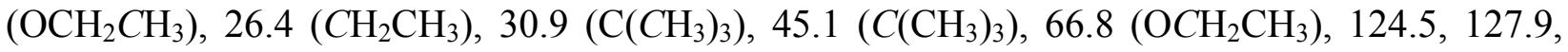
128.2, $146.9(\mathrm{Ar}-\mathrm{C}), 176.9(\mathrm{NCN}) ; \mathrm{IR} v / \mathrm{cm}^{-1}(\mathrm{ATR}): 1590 \mathrm{w}, 1500 \mathrm{~s}, 1195 \mathrm{~m}, 1168 \mathrm{~s}, 1153 \mathrm{~s}$, 1108m, 1028s, 894m, 862m, 809m, 780s, 759s; MS/EI m/z (\%): $470.3\left({ }^{\text {Dep }} \mathrm{NNNMg}^{+},<1\right), 447.4$ $\left({ }^{\text {Dep }} \mathrm{NNNH}^{+}, 14\right), 390.3\left({ }^{\mathrm{Dep}} \mathrm{NNNH}^{+}-\mathrm{Bu}^{t}, 73\right), 216.18\left(\mathrm{DepNCBu}^{t+}, 100\right)$. N.B. A reproducible microanalysis could not be obtained for the compound as it consistently co-crystallised with small amounts (ca. $4 \%$ ) of protonated ligand, ${ }^{\mathrm{Dep}} \mathrm{NNNH}$, which proved inseparable, even after several attempted fractional crystallizations.

\section{$\left({ }^{\text {Dip }} \mathrm{NNN}\right) \mathrm{MgI}\left(\mathrm{OEt}_{2}\right)(3 \mathrm{c})$}

Prepared using a similar procedure for the preparation of 3a, but using ${ }^{\text {Dip }} \mathrm{NNNH}(0.31 \mathrm{~g}, 0.61$ $\mathrm{mmol})$ and $\mathrm{MeMgI}(0.279 \mathrm{~mL}, 1.10 \mathrm{M}, 0.738 \mathrm{mmol})$ in diethyl ether. The title compound was obtained as an impure white solid after work-up. Several recrystallizations led to a small amount of the title compound as pure colorless crystalline solid (0.05 g, 9\%). M.p. $>200{ }^{\circ} \mathrm{C}$ (decomp.); 


\begin{abstract}
${ }^{1} \mathrm{H}$ NMR (400 MHz, $\left.298 \mathrm{~K}, \mathrm{C}_{6} \mathrm{D}_{6}\right): \delta 0.69$ (br, 6H, $\left.\mathrm{OCH}_{2} \mathrm{CH}_{3}\right), 1.10$ (s, 18H, C(CH3) $), 1.30$ (br, 24H, $\left.\mathrm{CH}\left(\mathrm{CH}_{3}\right)_{2}\right), 3.37$ (q, $\left.{ }^{3} \mathrm{~J}=7.0 \mathrm{~Hz}, 4 \mathrm{H}, \mathrm{OCH}_{2} \mathrm{CH}_{3}\right), 3.56\left(\mathrm{br}, 4 \mathrm{H}, \mathrm{CH}\left(\mathrm{CH}_{3}\right)_{2}, 7.05(\mathrm{~m}, 6 \mathrm{H}\right.$, $\mathrm{ArH}$ ); meaningful ${ }^{13} \mathrm{C}$ NMR data could not be obtained due to the low yield of pure material; IR $v / \mathrm{cm}^{-1}$ (ATR): 1502w, 1088s, 1015s, 895m, 794s, 755m, 700w, 684m; MS/EI m/z (\%): 728.8 $\left(\mathrm{M}^{+}, 100 \%\right)$; anal. calc. for $\mathrm{C}_{38} \mathrm{H}_{62} \mathrm{~N}_{3} \mathrm{MgIO}$ : C 62.68\%, $\mathrm{H} 8.58 \%$, N 5.77\%; found: $\mathrm{C} 62.50 \%, \mathrm{H}$ $8.64 \%$, N 5.54\%.
\end{abstract}

\title{
$\left\{\left({ }^{\mathrm{Mes}} \mathrm{NNN}\right) \mathrm{Ca}(\mathrm{THF})(\mu-\mathrm{I})\right\}_{2}(4)$
}

From one reaction of $\mathbf{2 a}$ with $\mathrm{CaI}_{2}$ in a THF solution carried out at $-80{ }^{\circ} \mathrm{C}$, and subsequently warmed to room temperature, a few colorless crystals of the title compound were isolated after work-up. No spectroscopic data for the compound could be obtained due to its very low yield.

\section{$\left({ }^{\mathrm{Mes}} \mathrm{NNN}\right)_{2} \mathrm{Mg}(\mathbf{5 a})$}

3a $(0.30 \mathrm{~g}, 0.47 \mathrm{mmol})$ was added to a Schlenk flask the bottom of which was coated with a sodium mirror (ca. $0.5 \mathrm{~g})$. The solid was dissolved in toluene $(20 \mathrm{~mL})$ and diethyl ether $(5 \mathrm{~mL})$, and the solution stirred for $1 \mathrm{hr}$. The solution was then filtered, and the resultant pale yellow filtrate concentrated in vacuo to the point of crystallization. The solution was then placed in the fridge overnight to yield the title compound as a colorless crystalline solid $(0.13 \mathrm{~g}, 24 \%) .{ }^{1} \mathrm{H}$ NMR (400 MHz, $\left.298 \mathrm{~K}, \mathrm{C}_{6} \mathrm{D}_{6}\right): \delta 1.18\left(\mathrm{~s}, 36 \mathrm{H}, \mathrm{C}\left(\mathrm{CH}_{3}\right)_{3}\right), 1.96\left(\mathrm{~s}, 24 \mathrm{H}, \mathrm{Ar}-\mathrm{CH}_{3}\right), 2.16(\mathrm{~s}, 12 \mathrm{H}$, Ar- $\left.\mathrm{CH}_{3}\right), 6.58(\mathrm{~s}, 8 \mathrm{H}, \mathrm{Mes}-H) ;{ }^{13} \mathrm{C}\left\{{ }^{1} \mathrm{H}\right\}$ NMR $\left(100 \mathrm{MHz}, 298 \mathrm{~K}, \mathrm{C}_{6} \mathrm{D}_{6}\right): \delta 20.1\left(\mathrm{Ar}-\mathrm{CH}_{3}\right), 20.8$

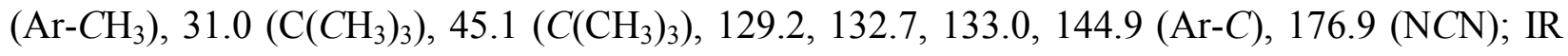
v/cm ${ }^{-1}$ (ATR): 1509s, 1165m, 1141s, 1021m, 957w, 935w, 877s, 852m, 758m; MS/EI m/z (\%): $860.9\left(\mathrm{M}^{+}, 3\right), 803.8\left(\mathrm{M}^{+}-\mathrm{Bu}^{t}, 3\right), 442.3\left({ }^{\mathrm{Mes}} \mathrm{NNNMg}^{+}, 3\right), 419.4\left({ }^{\mathrm{Mes}} \mathrm{NNN}^{+}, 8\right), 362.3\left({ }^{\mathrm{Mes}} \mathrm{NNN}^{+}-\right.$ 
$\left.\mathrm{Bu}^{t}, 60\right), 202.1\left(\mathrm{MesNCBu}^{t+}, 100\right)$. A reproducible microanalysis could not be obtained for the compound as it consistently co-crystallised with small amounts ( $c a .6 \%)$ of protonated ligand, ${ }^{\mathrm{Mes}} \mathrm{NNNH}$, which proved inseparable, even after several attempted fractional crystallizations.

\section{$\left({ }^{\mathrm{Dep}} \mathrm{NNN}\right)_{2} \mathrm{Mg}(\mathbf{5 b})$}

Prepared using a similar procedure for the preparation of 5a, but using $\mathbf{3 b}(0.67 \mathrm{~g}, 1.0 \mathrm{mmol})$ as the starting material. The title compound was obtained as a colorless crystalline solid after workup $(0.25 \mathrm{~g}, 54 \%)$. N.B. in one reduction reaction a few yellow crystals of the magnesium(I) dimer, $\left\{\left({ }^{\text {Dep }} \mathrm{NNN}\right) \mathrm{Mg}-\right\}_{2}$ 6, were obtained after work-up. No spectroscopic data could be obtained for this compound due to its low yield. Data for 5b: M.p. $>260{ }^{\circ} \mathrm{C} ;{ }^{1} \mathrm{H}$ NMR $(300$ $\left.\mathrm{MHz}, 298 \mathrm{~K}, \mathrm{C}_{6} \mathrm{D}_{6}\right): \delta 1.10\left(\mathrm{t},{ }^{3} J=7.5 \mathrm{~Hz}, 24 \mathrm{H}, \mathrm{CH}_{2} \mathrm{CH}_{3}\right), 1.16\left(\mathrm{~s}, 36 \mathrm{H}, \mathrm{C}\left(\mathrm{CH}_{3}\right)_{3}\right), 2.14\left(\mathrm{~m},{ }^{3} J=\right.$ $\left.7.5 \mathrm{~Hz}, 8 \mathrm{H}, \mathrm{CH}_{2} \mathrm{CH}_{3}\right), 2.56\left(\mathrm{~m},{ }^{3} \mathrm{~J}=7.5 \mathrm{~Hz}, 8 \mathrm{H}, \mathrm{CH}_{2} \mathrm{CH}_{3}\right), 6.95\left(\mathrm{~d},{ }^{3} \mathrm{~J}=7.3 \mathrm{~Hz}, 8 \mathrm{H}, \mathrm{ArH}\right), 7.03$ $\left(\mathrm{t},{ }^{3} J=7.3 \mathrm{~Hz}, 4 \mathrm{H}, \mathrm{Ar} H\right) ;{ }^{13} \mathrm{C}\left\{{ }^{1} \mathrm{H}\right\}$ NMR $\left(100 \mathrm{MHz}, 298 \mathrm{~K}, \mathrm{C}_{6} \mathrm{D}_{6}\right): \delta 13.0\left(\mathrm{CH}_{2} \mathrm{CH}_{3}\right), 24.0$

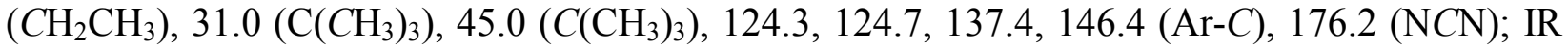
$v / \mathrm{cm}^{-1}$ (ATR): $1508 \mathrm{~m}, 1108 \mathrm{~m}, 1090 \mathrm{~m}, 1029 \mathrm{~m}, 995 \mathrm{~m}, 918 \mathrm{w}, 895 \mathrm{~m}, 862 \mathrm{w}, 810 \mathrm{w}, 781 \mathrm{~s}, 759 \mathrm{~s}$, 739m; MS/EI m/z (\%): $917.0\left(\mathrm{M}^{+}, 1\right), 859.9\left(\mathrm{M}^{+}-\mathrm{Bu}^{t}, 2\right), 470.3\left({ }^{\mathrm{Dep}} \mathrm{NNNMg}^{+}, 16\right), 216.18$ $\left(\mathrm{DepNC}^{\mathrm{Bu} t^{+}}, 100\right), 160.1$ (DepNC $\left.^{+}, 70\right)$; A reproducible microanalysis could not be obtained for the compound as it consistently co-crystallised with small amounts (ca. $8 \%$ ) of protonated ligand, ${ }^{\text {Dep }} \mathrm{NNNH}$, which proved inseparable, even after several attempted fractional crystallizations.

\section{$\left({ }^{\mathrm{Mes}} \mathrm{NNN}\right) \mathrm{BF}_{2}(7 \mathbf{a})$}

Freshly distilled $\mathrm{BF}_{3}\left(\mathrm{Et}_{2} \mathrm{O}\right)(0.77 \mathrm{~g}, 0.70 \mathrm{~mL}, 5.4 \mathrm{mmol})$ was added to a solution of $1 \mathrm{a}(2.10 \mathrm{~g}$, $4.0 \mathrm{mmol})$ in diethyl ether $(30 \mathrm{~mL})$ at room temperature. The reaction mixture was stirred 
overnight, resulting in a pale yellow solution. Volatiles were removed in vacuo and the residue extracted with toluene. Volatiles were removed from the extract in vacuo and the residue heated at $40{ }^{\circ} \mathrm{C}$ under reduced pressure for $1 \mathrm{~h}$. This yielded the title compound as pale yellow solid (1.80 g, 96\%). Crystals of the compound suitable for X-ray crystallography were grown from a toluene solution. M.p. $164-166{ }^{\circ} \mathrm{C} ;{ }^{1} \mathrm{H}$ NMR $\left(\mathrm{C}_{6} \mathrm{D}_{6}, 400 \mathrm{MHz}, 298 \mathrm{~K}\right): \delta 1.17\left(\mathrm{~s}, 18 \mathrm{H}, \mathrm{C}\left(\mathrm{CH}_{3}\right)_{3}\right)$, 2.03 (s, 6H, Ar- $\left.\mathrm{CH}_{3}\right), 2.37$ (s, 12H, Ar- $\left.\mathrm{CH}_{3}\right), 6.69$ (s, 4H, $\left.\mathrm{ArH}\right) ;{ }^{13} \mathrm{C}\left\{{ }^{1} \mathrm{H}\right\} \mathrm{NMR}\left(\mathrm{C}_{6} \mathrm{D}_{6}, 101 \mathrm{MHz}\right.$, $298 \mathrm{~K}): \delta 19.7\left(\mathrm{Ar}-\mathrm{CH}_{3}\right), 20.9\left(\mathrm{Ar}-\mathrm{CH}_{3}\right), 30.4\left(\mathrm{C}\left(\mathrm{CH}_{3}\right)_{3}\right), 42.4\left(C\left(\mathrm{CH}_{3}\right)_{3}\right), 129.2,135.8,136.8$, 137.1 (Ar-C), $175.6(\mathrm{NCN}) ;{ }^{11} \mathrm{~B}$ NMR $\left(\mathrm{C}_{6} \mathrm{D}_{6}, 128 \mathrm{MHz}, 298 \mathrm{~K}\right): \delta 0.7\left(\mathrm{t}, J_{\mathrm{BF}}=28 \mathrm{~Hz}\right) ;{ }^{19} \mathrm{~F} \mathrm{NMR}$ $\left(\mathrm{C}_{6} \mathrm{D}_{6}, 376 \mathrm{MHz}, 298 \mathrm{~K}\right): \delta-134.3$ (q, $J_{\mathrm{BF}}=28 \mathrm{~Hz}$ ); IR $v / \mathrm{cm}^{-1}$ (ATR): $1609 \mathrm{w}, 1530 \mathrm{~s}, 1515 \mathrm{~s}$, 1151w, 1130w, 1079m, 1047s, 1029s, 976s, 942s, 875w, 850m, 800w; MS/EI m/z (\%): 467.4 $\left(\mathrm{M}^{+}, 10\right), 452.4\left(\mathrm{M}^{+}-\mathrm{Me}, 27\right), 362.3\left({ }^{\mathrm{Mes} N N N}-\mathrm{Bu}^{t+}, 22\right), 202.1$ (MesNCBu$\left.{ }^{t}, 100\right)$; anal. calc. for $\mathrm{C}_{30} \mathrm{H}_{44} \mathrm{BF}_{2} \mathrm{~N}_{3}$ : C 71.94\%, H 8.63\%, N 8.99\%; found: C 70.84\%, H 8.78\%, N 9.05\%.

\section{$\left({ }^{\mathrm{Dep}} \mathrm{NNN}\right) \mathrm{BF}_{2}(\mathbf{7 b})$}

Prepared using a similar procedure for the preparation of 7a, but using $\mathbf{1 b}(0.50 \mathrm{~g}, 0.95 \mathrm{mmol})$ and $\mathrm{BF}_{3}\left(\mathrm{OEt}_{2}\right)(0.18 \mathrm{~g}, 0.16 \mathrm{~mL}, 1.29 \mathrm{mmol})$. The title compound was obtained as pale yellow solid after work-up. Crystals of the compound suitable for X-ray crystallography were grown from a hexane solution (0.42 g, 89 \%). M.p. $160-161{ }^{\circ} \mathrm{C} ;{ }^{1} \mathrm{H}$ NMR $\left(\mathrm{C}_{6} \mathrm{D}_{6}, 400 \mathrm{MHz}, 298 \mathrm{~K}\right): \delta$ $1.13\left(\mathrm{~s}, 18 \mathrm{H}, \mathrm{C}\left(\mathrm{CH}_{3}\right)_{3}\right), 1.27\left(\mathrm{t},{ }^{3} \mathrm{~J}=7.5 \mathrm{~Hz}, 12 \mathrm{H}, \mathrm{CH}_{2} \mathrm{CH}_{3}\right), 2.53\left(\mathrm{~m}, 4 \mathrm{H}, \mathrm{CH}_{2} \mathrm{CH}_{3}\right), 3.00(\mathrm{~m}, 4 \mathrm{H}$, $\left.\mathrm{CCH}_{2} \mathrm{CH}_{3}\right), 7.00\left(\mathrm{~d},{ }^{3} \mathrm{~J}=7.6 \mathrm{~Hz}, 4 \mathrm{H}, \mathrm{Ar} H\right), 7.08\left(\mathrm{dd},{ }^{3} J=8.5 \mathrm{~Hz},{ }^{3} J=6.6 \mathrm{~Hz}, 2 \mathrm{H}, \mathrm{Ar} H\right) ;{ }^{13} \mathrm{C}\left\{{ }^{1} \mathrm{H}\right\}$

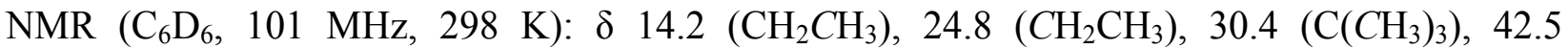
$\left(C\left(\mathrm{CH}_{3}\right)_{3}\right), 125.6,127.9,138.1,141.4(\mathrm{Ar}-\mathrm{C}), 175.7(\mathrm{NCN}) ; \mathrm{IR} \mathrm{v} / \mathrm{cm}^{-1}(\mathrm{ATR}): 1648 \mathrm{w}, 1529 \mathrm{~m}$, 1512m, 1134w, 1093m, 1048s, 971s, 935w, 885m, 874m, 811m, 775s; MS/EI m/z (\%): 495.6 $\left(\mathrm{M}^{+}, 6\right), 466.5\left(\mathrm{M}^{+}\right.$-Et, 36), $438.4\left(\mathrm{M}^{+}-\mathrm{Bu}^{t}, 4\right), 216.2\left(\mathrm{DepNCBu}^{t_{+}}, 100\right), 160.1$ (DepNC $\left.{ }^{+}, 76\right)$. 


\begin{abstract}
N.B. A reproducible microanalysis could not be obtained due to the moisture sensitive nature of the compound.
\end{abstract}

\title{
$\operatorname{PhC}\left\{\mathrm{C}\left(\mathrm{NEt}_{2}\right) \mathrm{N}(\mathrm{Mes})\right\}_{2} \mathrm{BF}_{2}(8)$
}

Freshly distilled $\mathrm{BF}_{3}\left(\mathrm{Et}_{2} \mathrm{O}\right)(0.062 \mathrm{~g}, 0.054 \mathrm{~mL}, 0.43 \mathrm{mmol})$ was added to a solution of $\left[\mathrm{PhC}\left\{\mathrm{C}\left(\mathrm{NEt}_{2}\right) \mathrm{N}(\mathrm{Mes})\right\}_{2}\right] \mathrm{Li}(0.230 \mathrm{~g}, 0.43 \mathrm{mmol})$ in diethyl ether $(5 \mathrm{~mL})$ at room temperature. The solution was then stirred at room temperature for $16 \mathrm{hr}$. Volatiles were removed in vacuo and hexane added to the residue. This resulted in a yellow solution containing a white suspended solid. The suspension was filtered and volatiles removed in vacuo, leaving the title compound as a pale yellow solid (160 mg, 78 \%). M.p. $130-135{ }^{\circ} \mathrm{C} ;{ }^{1} \mathrm{H}$ NMR $\left(\mathrm{C}_{6} \mathrm{D}_{6}, 400 \mathrm{MHz}, 298 \mathrm{~K}\right): \delta 0.42$ $\left(\mathrm{t},{ }^{3} \mathrm{~J}=7.2 \mathrm{~Hz}, 12 \mathrm{H}, \mathrm{NCH}_{2} \mathrm{CH}_{3}\right), 2.15\left(\mathrm{~s}, 6 \mathrm{H}, \mathrm{Ar}-\mathrm{CH}_{3}\right), 2.54\left(\mathrm{~s}, 12 \mathrm{H}, \mathrm{Ar}-\mathrm{CH}_{3}\right), 2.82$ (q, ${ }^{3} J=7.2$ $\left.\mathrm{Hz}, 8 \mathrm{H}, \mathrm{NCH}_{2} \mathrm{CH}_{3}\right), 6.81(\mathrm{~s}, 4 \mathrm{H}, \mathrm{Ar} H), 7.07\left(\mathrm{~d},{ }^{3} \mathrm{~J}=7.6 \mathrm{~Hz}, 1 \mathrm{H}, \mathrm{PhH}\right), 7.13\left(\mathrm{t},{ }^{3} J=7.6 \mathrm{~Hz}, 2 \mathrm{H}\right.$, $\mathrm{Ph} H), 7.47\left(\mathrm{~d},{ }^{3} J=7.6 \mathrm{~Hz}, 2 \mathrm{H}, \mathrm{Ph} H\right) ;{ }^{13} \mathrm{C}\left\{{ }^{1} \mathrm{H}\right\} \quad \mathrm{NMR}\left(\mathrm{C}_{6} \mathrm{D}_{6}, 75 \mathrm{MHz}, 298 \mathrm{~K}\right): \delta 12.0$ $\left(\mathrm{NCH}_{2} \mathrm{CH}_{3}\right), 13.2\left(\mathrm{Ar}-\mathrm{CH}_{3}\right), 20.0\left(\mathrm{Ar}-\mathrm{CH}_{3}\right), 44.1\left(\mathrm{NCH}_{2} \mathrm{CH}_{3}\right), 126.3,128.9,129.7,134.4,135.0$, 135.2, 139.5, $140.0(\mathrm{Ar}-\mathrm{C}), 168.8(\mathrm{NCN})$, N.B. $\mathrm{C} C(\mathrm{Ph}) \mathrm{C}$ resonance not observed; ${ }^{11} \mathrm{~B}$ NMR $\left(\mathrm{C}_{6} \mathrm{D}_{6}, 128 \mathrm{MHz}, 298 \mathrm{~K}\right): \delta 1.4\left(\mathrm{t}, J_{\mathrm{BF}}=30 \mathrm{~Hz}\right) ;{ }^{19} \mathrm{~F}$ NMR $\left(\mathrm{C}_{6} \mathrm{D}_{6}, 376 \mathrm{MHz}, 298 \mathrm{~K}\right): \delta-144.2(\mathrm{q}$, $J_{\mathrm{BF}}=30 \mathrm{~Hz}$ ); IR $v / \mathrm{cm}^{-1}$ (ATR): $1611 \mathrm{w}, 1596 \mathrm{w}, 1500 \mathrm{~m}, 1229 \mathrm{~m}, 1134 \mathrm{~m}, 1056 \mathrm{~s}, 998 \mathrm{~m}, 925 \mathrm{~m}$, 849m, 804m, 773m; MS/EI m/z (\%): $572.5\left(\mathrm{M}^{+}, 31\right), 557.4\left(\mathrm{M}^{+}-\mathrm{Me}, 64\right), 543.4\left(\mathrm{M}^{+}-\mathrm{Et}, 27\right)$, $451.3\left(\mathrm{PhC}\left\{\mathrm{C}\left(\mathrm{NEt}_{2}\right) \mathrm{N}(\mathrm{Mes})\right\}_{2}-\mathrm{NEt}_{2}{ }^{+}\right.$, 95). N.B. A reproducible microanalysis could not be obtained due to the moisture sensitive nature of the compound.

\section{$\mathrm{HC}\left\{\mathrm{C}\left(\mathrm{NEt}_{2}\right) \mathrm{N}(\mathrm{Mes})\right\}_{2} \mathrm{AICl}_{2}$ (10)}

From one reaction of $\left[\mathrm{HC}\left\{\mathrm{C}\left(\mathrm{NEt}_{2}\right) \mathrm{N}(\mathrm{Mes})\right\}_{2}\right] \mathrm{Li}$ with $\mathrm{Al}^{\mathrm{I}} \mathrm{Cl}$ in a toluene/THF solution carried out at $-80{ }^{\circ} \mathrm{C}$, and subsequently warmed to room temperature, a few colorless crystals of the title 
compound were isolated after work-up. No spectroscopic data for the compound could be obtained due to its very low yield.

\section{$\mathrm{HC}\left\{\mathrm{C}\left(\mathrm{NEt}_{2}\right) \mathrm{N}(\mathrm{Mes})\right\}_{2} \mathrm{Ga}(\mathrm{H}) \mathrm{Cl}(11)$}

From one reaction of $\left[\mathrm{HC}\left\{\mathrm{C}\left(\mathrm{NEt}_{2}\right) \mathrm{N}(\mathrm{Mes})\right\}_{2}\right] \mathrm{Li}$ with $\mathrm{Ga}^{\mathrm{I}} \mathrm{Cl}$ in a toluene/THF solution carried out at $-80{ }^{\circ} \mathrm{C}$, and subsequently warmed to room temperature, a few colorless crystals of the title compound were isolated after work-up. No spectroscopic data for the compound could be obtained due to its very low yield.

X-Ray Crystallography. Crystals of ${ }^{A r} \mathrm{NNNH}, \mathbf{1 c}$, 3a-c, 4, 5a-b, 6, 7a-b, 8, 10 and 11 suitable for X-ray structural determination were mounted in silicone oil. Crystallographic measurements were made using either a Bruker Apex X8 diffractometer employing a graphite monochromator with Mo K $\alpha$ radiation $(\lambda=0.71073 \AA)$, or the MX1 beamline of the Australian Synchrotron $(\lambda=$ $0.71080 \AA$ or $0.71090 \AA$ ). The software package Blu-Ice ${ }^{9}$ was used for synchrotron data acquisition, while the program $\mathrm{XDS}^{10}$ was employed for synchrotron data reduction. The structures were solved by direct methods and refined on $\mathrm{F}^{2}$ by full matrix least squares $\left(\right.$ SHELX $\left.97^{11}\right)$ using all unique data. All non-hydrogen atoms are anisotropic with non-hydride and non-amine hydrogen atoms included in calculated positions (riding model). The positional parameters of the hydride ligand of $\mathbf{1 1}$ and the amine protons of ${ }^{\mathrm{Ar}} \mathrm{NNNH}$ were refined. Compound 6 co-crystallized with a small amount (ca. 15\%) of the hydrolysis product $\left\{\left({ }^{\text {Dep }} \mathrm{NNN}\right) \mathrm{Mg}(\mu-\mathrm{OH})\right\}_{2}$. Crystal data, details of data collections and refinement are given in Table S1 of the Supplementary Material. 


\section{Results and Discussion}

Ligand development. Three new 1,3,5-triazapentadiene pro-ligands, ${ }^{\mathrm{Ar}} \mathrm{NNNH}(\mathrm{Ar}=\mathrm{Mes}$ (mesityl), Dep (2,6-diethylphenyl) or Dip (2,6-diisopropylphenyl)), bearing bulky $N$-aryl and tert-butyl backbone substituents, were synthesized using a variation of previously reported methods. That is, the appropriate imidoyl chloride and amidine were combined and heated in a melt at $200-230{ }^{\circ} \mathrm{C}$ for several hours, before basic work-up (Scheme 1). This route reliably afforded good yields of ${ }^{\mathrm{Mes}} \mathrm{NNNH}$ and ${ }^{\mathrm{Dep}} \mathrm{NNNH}$, though the synthesis of ${ }^{\mathrm{Dip}} \mathrm{NNNH}$ was poorly reproducible, and always led to mixtures of inseparable products, whether ${ }^{\text {Dip }} \mathrm{NNNH}$ was present in the mix or not. Alternative procedures for the synthesis of ${ }^{\text {Dip }} \mathrm{NNNH}$ were explored, though none were successful.

The spectroscopic data for ${ }^{\mathrm{Mes}} \mathrm{NNNH}$ and ${ }^{\mathrm{Dep}} \mathrm{NNNH}$ are consistent with their proposed structures, and suggest the compounds exist as one isomeric form in solution. In contrast, these data for ${ }^{\text {Dip }} \mathrm{NNNH}$ could not be confidently assigned as a result of the inability to obtain the compound in a pure form. However, the X-ray crystal structures of all three pro-ligands were obtained (see Figure 2 for the structure of ${ }^{\text {Dep }} \mathrm{NNNH}$, and Supplementary Material for structures of ${ }^{\mathrm{Mes}} \mathrm{NNNH}$ and ${ }^{\mathrm{Dip}} \mathrm{NNNH}$ ). In all, the $\mathrm{N}_{3} \mathrm{C}_{2}$ backbone is non-planar, and the amine proton resides on one of the terminal $N$-centers. This is a similar situation to the majority of the handful of $N$-aryl substituted 1,3,5-triazapentadienes that have been crystallographically characterized. $^{4 \mathrm{~b}, 12}$ As in those cases, the N-C bond lengths in the non-planar, unconjugated backbone suggest alternating localized double and single bonds. The only previously reported exception is the related compound, $\mathrm{N}\{\mathrm{C}(\mathrm{Ph})=\mathrm{N}(\mathrm{Dip})\}\{\mathrm{C}(\mathrm{Ph})-\mathrm{N}(\mathrm{H})(\mathrm{Dip})\}$, in which the N-C bonds within the $\mathrm{N}_{3} \mathrm{C}_{2}$ backbone are conjugated, and which exhibits intramolecular $\mathrm{N}-\mathrm{H} \cdots \mathrm{N}$ hydrogen bonding, as is typically seen for related $\beta$-diketimines. ${ }^{4 \mathrm{~b}}$ 

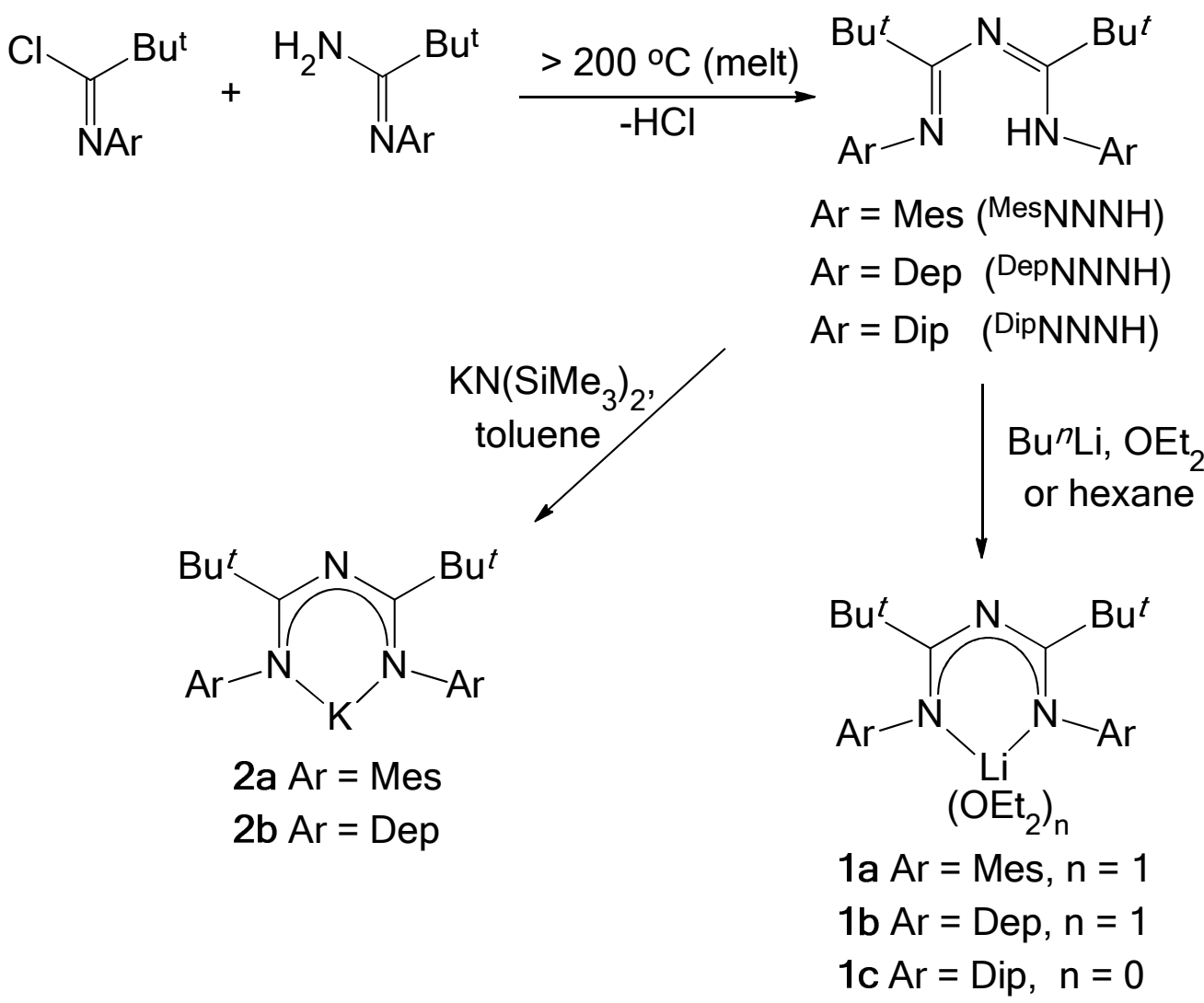

Scheme 1. Synthesis of new triazapentadienes and compounds $\mathbf{1 a - c}$ and $\mathbf{2 a - b}$.

The deprotonation of the ${ }^{\mathrm{Ar}} \mathrm{NNNH}$ with $\mathrm{Bu}^{n} \mathrm{Li}$ proceeded smoothly, yielding 1,3,5triazapentadienyl lithium salts, 1a-c, which were seen as suitable ligand transfer reagents for further chemistry (Scheme 1). It is worth noting that lithiation of impure mixtures containing ${ }^{\text {Dip }} \mathrm{NNNH}$ allowed for the separation of the pure lithium salt, 1c, upon work-up. Deprotonation of ${ }^{\mathrm{Mes}} \mathrm{NNNH}$ and ${ }^{\text {Dep }} \mathrm{NNNH}$ with $\mathrm{KN}\left(\mathrm{SiMe}_{3}\right)_{2}$ to give triazapentadienyl potassium salts, $\mathbf{2 a - b}$, is also achievable, though the yields are lower than those obtained from the corresponding lithiation reactions.

The X-ray crystal structure of 1c (see Figure 2) was obtained, and this revealed it to be monomeric, with the Li center $N, N$-chelated by a near planar, delocalized 1,3,5-triazapentadienyl 
ligand, as has been previously observed for lithium salts of this ligand class. ${ }^{12 \mathrm{~d}}$ However, in the present case, the Li center is two-coordinate and unsolvated, whereas in previously reported 1,3,5-triazapentadienyl lithium complexes, and $\mathbf{1 a - b}$, the lithium center is coordinated by an ether molecule. This presumably reflects the extreme steric bulk of the ligand in $\mathbf{1 c}$, the backbone tertbutyl groups of which interact with the Dip groups, compressing the bite angle of the chelating ligand, relative to those in less bulky 1,3,5-triazapentadienyl lithium complexes. It is of note that related two-coordinate $N, N^{\prime}$-chelated $\beta$-diketiminato lithium complexes have been previously described. $^{13}$

(a)

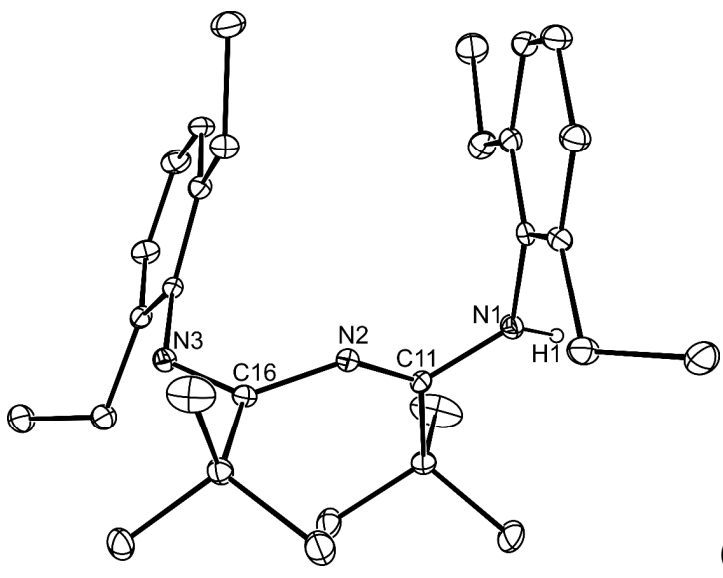

(b)

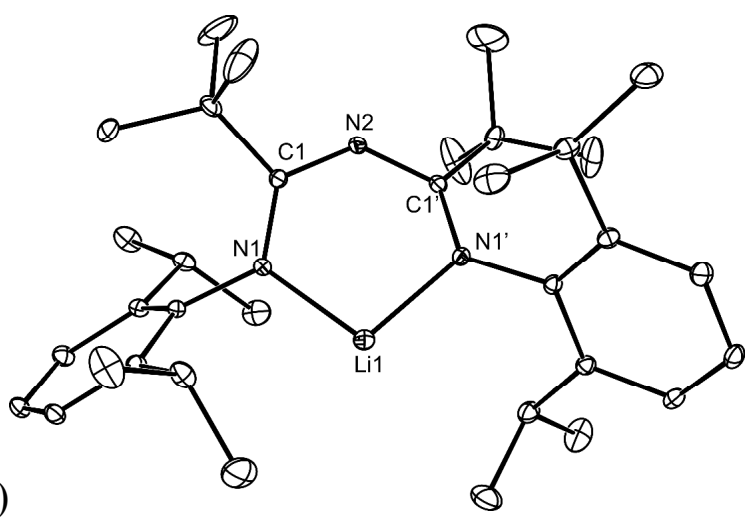

Fig. 2. Thermal ellipsoid plot (25\% probability surface) of the molecular structures of (a) ${ }^{\text {Dep }} \mathrm{NNNH}$, and (b) $\left({ }^{\mathrm{Dip}} \mathrm{NNN}\right) \mathrm{Li}$ 1c. Hydrogen atoms, except the amine proton, are omitted. Selected bond lengths $(\AA)$ and angles $\left(^{\circ}\right)$ for ${ }^{\mathrm{Dep}} \mathrm{NNNH}$ : N(1)-C(11) 1.375(3), N(2)-C(11) 1.274(3), N(2)-C(16) 1.384(3), N(3)-C(16) 1.290(3); for 1c: $\mathrm{N}(1)-\mathrm{Li}(1)$ 1.883(2), N(1)-C(1) 1.3227(15), C(1)-N(2) 1.3393(14), N(1)-Li(1)-N(1)' 102.33(16). 
Group 2 chemistry. We have had considerable success in preparing magnesium(I) dimers stabilized by incorporation of bulky $N, N$-chelating $\beta$-diketiminate ligands. ${ }^{14}$ Given the structural similarities between $\beta$-diketiminate and 1,3,5-triazapentadienyl ligands, and the potentially higher electron density at the $N$-donor centers of the latter, we believed that bulky triazapentadienyls might be suitable for the preparation of new magnesium(I) dimers. Moreover, it was deemed worthwhile to explore their use in the preparation of related calcium(I) dimers, $\mathrm{LCa}-\mathrm{CaL}$, examples of which are currently unknown.

As suitable precursors to target magnesium(I) dimers, the magnesium(II) iodide complexes, 3a-b, were prepared in good yields by reaction of the appropriate pro-ligand with MeMgI (Scheme 2). The more sterically hindered complex, 3c, was obtained by a similar procedure, but the impurity of the triazapentadiene starting material, ${ }^{\text {Dip }} \mathrm{NNNH}$, meant that only low yields of pure $\mathbf{3 c}$ could be obtained after work-up. The magnesium compounds are also accessible by reaction of $\mathrm{MgI}_{2}$ with lithium triazapentadienyls, 1a-c, though the product yields are much reduced. For sake of comparison, reactions of the potassium salts, $\mathbf{2 a - b}$, with $\mathrm{CaI}_{2}$ were carried out, though these typically led to intractable product mixtures. With that said, from one reaction, a few crystals of the triazapentadienyl calcium iodide complex, $\mathbf{4}$, were isolated. Although no spectroscopic data could be obtained for this compound, details of its X-ray crystal structure are included below. 

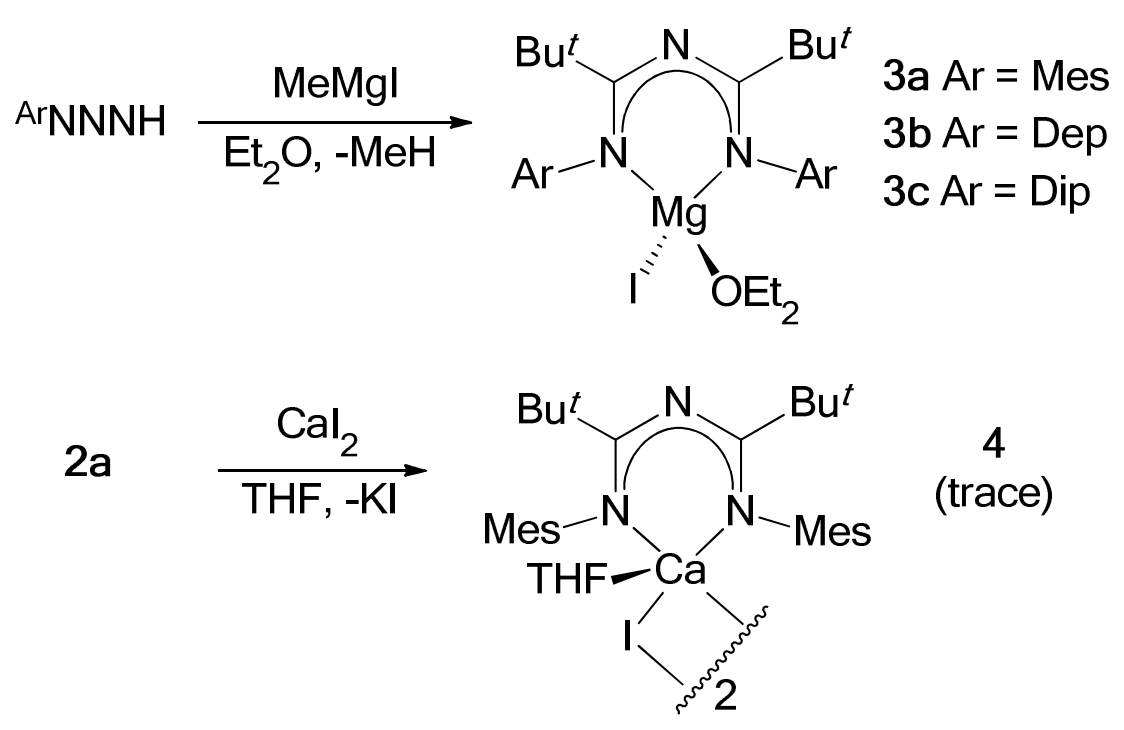

Scheme 2. Synthesis of compounds 3a-c and 4.

The molecular structures of $\mathbf{3 a - c}$ are broadly similar and so only that for $\mathbf{3 b}$ is depicted in Figure 3 (see Supplementary Material for structures of $\mathbf{3 a}$ and $\mathbf{3 c}$ ). All the compounds are monomeric with distorted tetrahedral $\mathrm{Mg}$ centres. Presumably due to the bulk of the triazapentadienyl ligands, and the resultant steric buttressing with the $\mathrm{MgI}\left(\mathrm{OEt}_{2}\right)$ fragments of the complexes, the $\mathrm{N}_{3} \mathrm{C}_{2}$ backbones of those ligands are slightly distorted (by twisting) from being planar, while the Mg centers sit significantly above the backbone least squares plane (e.g. by 0.82 $\AA$ in the case of $\mathbf{3 b}$ ). Despite this, the N-C bond lengths within those backbones are suggestive of partial delocalization. By comparison, a greater degree of electronic delocalisation is typically seen in related $\beta$-diketiminate complexes, (Nacnac) $\mathrm{MgI}\left(\mathrm{OEt}_{2}\right)$, the $\mathrm{N}_{2} \mathrm{C}_{3}$ backbone fragments of which are essentially planar, ${ }^{15}$ even though their $\mathrm{Mg}$ centers can sit significantly above that plane (cf. 3a-c). Not surprisingly, due to the relative size of calcium versus magnesium (covalent radii: $1.74 \AA$ and $1.36 \AA$ respectively $^{16}$ ), the calcium iodide complex, 4, exists as an iodide bridged dimer in the solid state (Figure 3). The calcium centers of the complex have distorted square based pyramidal coordination geometries, and are markedly above the ligand backbone least 
squares plane (by $1.39 \AA$ ). Moreover, as is the case for 3a-c, the $\mathrm{N}_{3} \mathrm{C}_{2}$ backbones of $\mathbf{4}$ are slightly distorted from planarity, though they seemingly exhibit significant electronic delocalization.

(a)

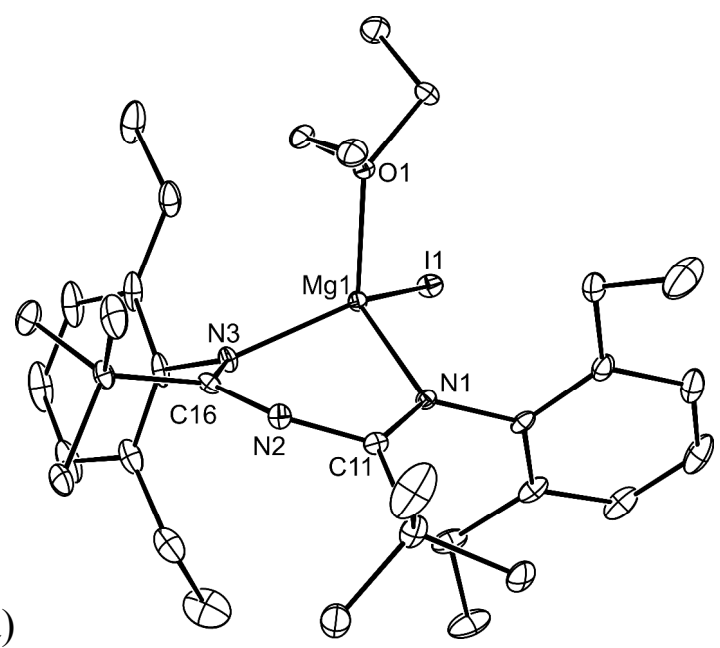

(b)

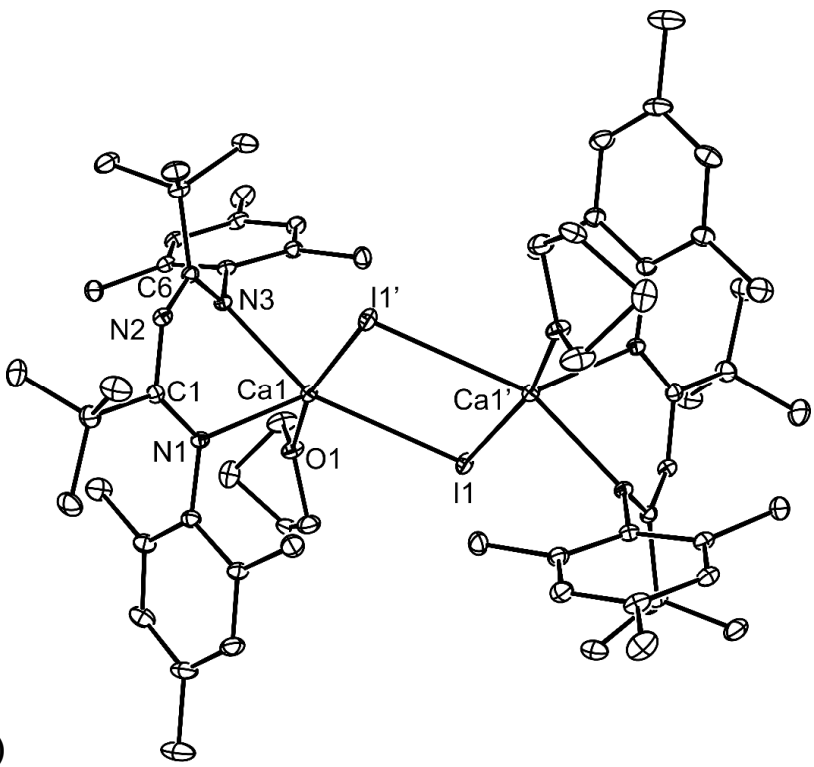

Fig. 3. Thermal ellipsoid plot (25\% probability surface) of the molecular structures of (a) $\left({ }^{\mathrm{Dep}} \mathrm{NNN}\right) \mathrm{MgI}\left(\mathrm{OEt}_{2}\right) \mathbf{3 b}$, and (b) $\left\{\left({ }^{\mathrm{Mes}} \mathrm{NNN}\right) \mathrm{Ca}(\mathrm{THF})(\mu-\mathrm{I})\right\}_{2}$ 4. Hydrogen atoms are omitted. Selected bond lengths $(\AA)$ and angles $\left(^{\circ}\right)$ for 3b: $\mathrm{I}(1)-\mathrm{Mg}(1)$ 2.6629(8), $\mathrm{Mg}(1)-\mathrm{O}(1)$ 2.0277(19), $\mathrm{Mg}(1)-\mathrm{N}(3)$ 2.029(2), $\mathrm{Mg}(1)-\mathrm{N}(1)$ 2.048(2), N(1)-C(11) 1.305(3), N(2)-C(16) 1.335(3), N(2)$\mathrm{C}(11)$ 1.362(3), N(3)-C(16) 1.344(3), N(3)-Mg(1)-N(1) 92.02(9); for 4: I(1)-Ca(1) 3.1039(12), I(1)-Ca(1)' 3.1471(10), Ca(1)-N(3) 2.330(2), Ca(1)-N(1) 2.332(2), Ca(1)-O(1) 2.354(2), N(1)$\mathrm{C}(1)$ 1.309(3), $\mathrm{C}(1)-\mathrm{N}(2)$ 1.359(3), N(2)-C(6) 1.340(3), N(3)-C(6) 1.327(3), N(3)-Ca(1)-N(1) 76.93(8).

Reductions of the magnesium iodide complexes, $\mathbf{3 a}$ and $\mathbf{3 b}$, over sodium mirrors (excess) in toluene/diethyl ether solutions were carried out at room temperature. In the former case, all of the magnesium iodide starting material was consumed within an hour, and a number of products 
were formed, as determined by a ${ }^{1} \mathrm{H}$ NMR spectroscopic analysis of the product mixture. The reduction of bulkier $\mathbf{3 b}$ was slower, but was still complete within 24 hours, again yielding a mixture of products. The reactions of $\mathbf{3 a - b}$ with sodium mirrors are markedly faster than those reported for the sterically similar $N$-Mes and $N$-Dep substituted (Nacnac)MgI(OEt 2 ) complexes (bearing methyl backbone substituents ${ }^{15}$ ). These take $c a .4-5$ days to reach completion and afford good yields of magnesium(I) dimers, (Nacnac)Mg-Mg(Nacnac), and small amounts of homoleptic magnesium(II) by-products, $\mathrm{Mg}(\mathrm{Nacnac})_{2}$. In the present reactions, the only products that could be isolated in appreciable quantities were the related colorless, crystalline homoleptic complexes, 5a-b (Scheme 3). It should be noted, however, that in one reduction of $\mathbf{3 b}$, a few yellow crystals of the triazapentadienyl coordinated magnesium(I) dimer, 6 , were isolated and crystallographically characterized (see below). Unfortunately, no spectroscopic data could be obtained for the compound due to its very low yield. It is currently not know why triazapentadienyl magnesium iodide complexes are considerably more reactive towards reduction than their $\beta$-diketiminato counterparts, though this is a possible reason why homoleptic complexes are predominantly formed from reduction of the former. That is, the prevalence of transient magnesium(I) dimer products to undergo disproportionation processes under the reaction conditions employed, is greater for triazapentadienyl based systems. With that said, altering the reduction conditions by treating 3a-b with stoichiometric amounts of sodium or $\mathrm{KC}_{8}$, still led to $\mathbf{5 a - b}$ as the major isolated products. 


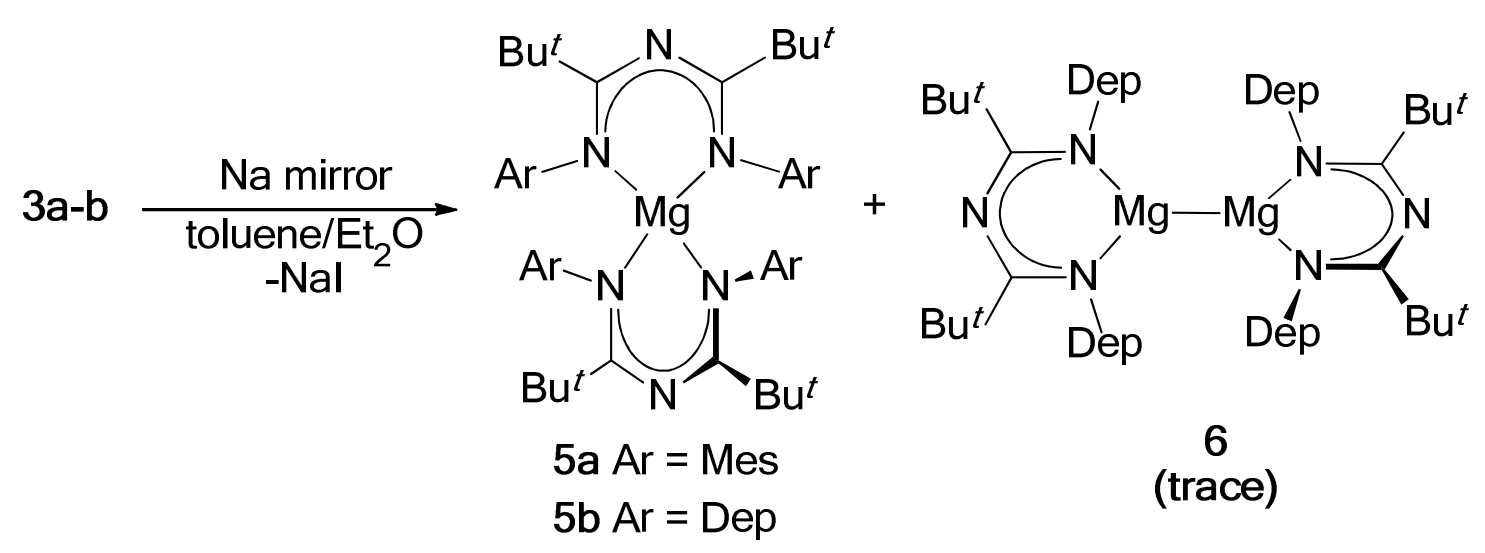

Scheme 3. Synthesis of compounds 5a-b and 6.

The crystal structures of $\mathbf{5 a}$ and $\mathbf{5 b}$ are very similar so only the molecular structure of $\mathbf{5 a}$ is depicted in Figure 4 (see Supplementary Material for that of $\mathbf{5 b}$ ). The compounds possess distorted tetrahedral magnesium geometries and overall the structures are closely related to those of homoleptic $\beta$-diketiminato magnesium complexes, $\mathrm{Mg}(\mathrm{Nacnac})_{2}$, including comparable $\mathrm{N}-\mathrm{Mg}$ bond lengths and N-Mg-N angles. ${ }^{14}$ The molecular structure of the magnesium(I) dimer, 6 , reveals it again to be broadly similar to its $\beta$-diketiminato analogues, ${ }^{14}$ and its $\mathrm{Mg}$-Mg bond length lies in the range recorded for those compounds. The magnesium centers of the compound have trigonal planar coordination geometries and its six-membered heterocycles are close to planar and exhibit N-C bond lengths that imply significant electronic delocalization over the ligand backbone fragments. 

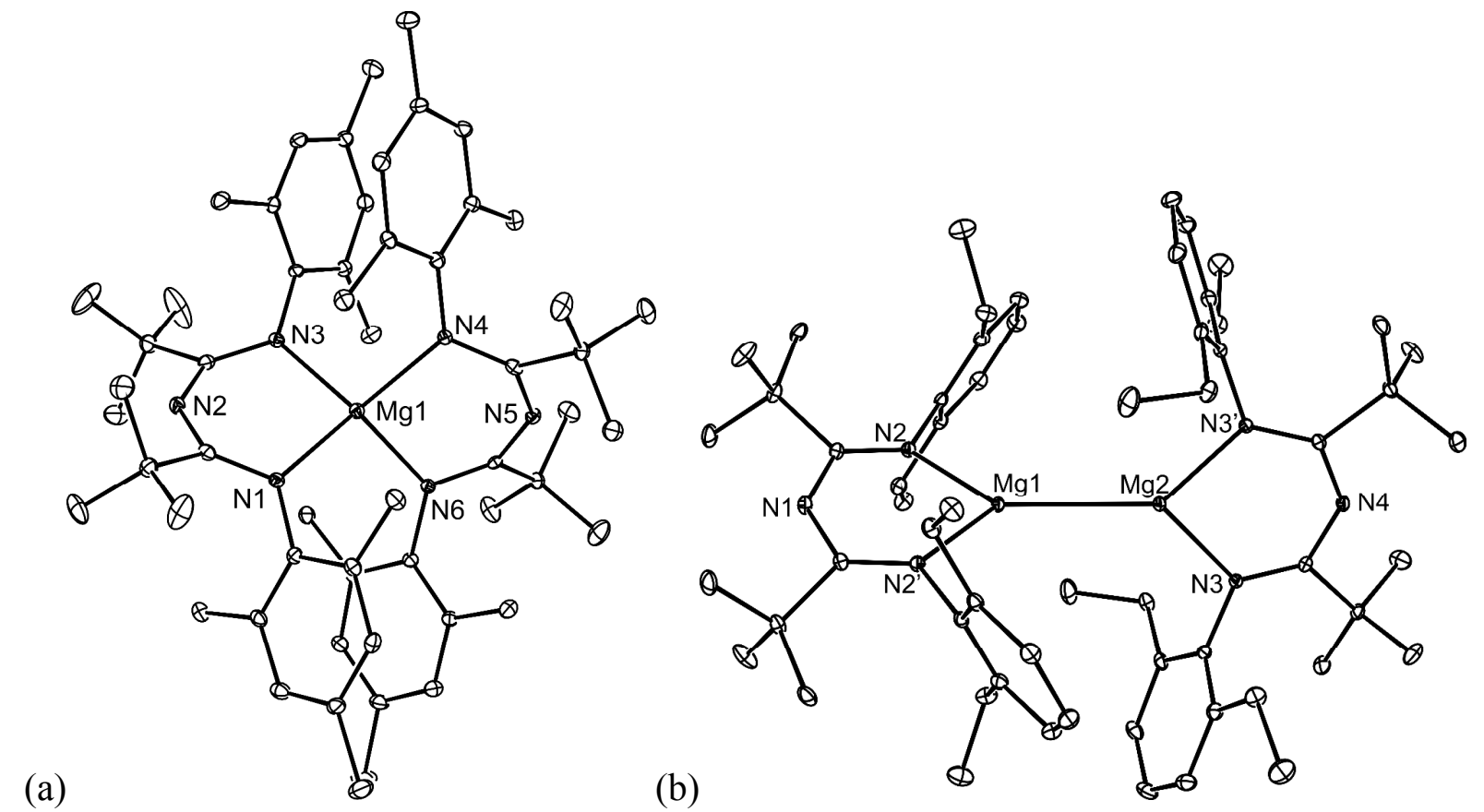

Fig. 4. Thermal ellipsoid plot (25\% probability surface) of the molecular structures of (a) $\left({ }^{\mathrm{Mes}} \mathrm{NNN}\right)_{2} \mathrm{Mg}$ 5a, and (b) $\left\{\left({ }^{\mathrm{Dep}} \mathrm{NNN}\right) \mathrm{Mg}-\right\}_{2}$ 6. Hydrogen atoms are omitted. Selected bond lengths $(\AA)$ and angles $\left(^{\circ}\right)$ for 5a: $\operatorname{Mg}(1)-\mathrm{N}(6)$ 2.083(3), $\operatorname{Mg}(1)-\mathrm{N}(3)$ 2.089(3), $\operatorname{Mg}(1)-\mathrm{N}(1)$ 2.102(3), $\mathrm{Mg}(1)-\mathrm{N}(4)$ 2.121(3), N(3)-Mg(1)-N(1) 93.02(12), N(6)-Mg(1)-N(4) 93.31(11); for 6: $\operatorname{Mg}(1)-\operatorname{Mg}(2)$ 2.8576(11), $\operatorname{Mg}(1)-\mathrm{N}(2)$ 2.0638(13), $\mathrm{Mg}(2)-\mathrm{N}(3)$ 2.0634(13), N(2)-Mg(1)-N(2)' 87.75(7), N(3)-Mg(2)-N(3)' 89.74(7).

Group 13 chemistry. $\beta$-diketiminates have proved their worth as stabilizing ligands in low oxidation state group 13 chemistry, and metal(I) complexes of the type (Nacnac)M: $(\mathrm{M}=\mathrm{Al}, \mathrm{Ga}$, In or $\mathrm{Tl}$ ), are known for all the heavier elements. ${ }^{1 \mathrm{~b}, 17}$ There are, however, no known examples of related boron(I) heterocycles incorporating these ligands, which computational studies attribute to very narrow singlet-triplet gaps in those systems. ${ }^{18}$ This would make them highly susceptible to, for example, intramolecular $\mathrm{C}-\mathrm{H}$ bond activation processes. We wondered whether substituting $\beta$-diketiminates with more electron rich ligands might help stabilize six-membered boron(I) 
heterocycles. In order to access potential precursors to such targets, $\mathrm{BF}_{3}\left(\mathrm{OEt}_{2}\right)$ was treated with the triazapentadienyl lithium salts, 1a-b, to give excellent isolated yields of the boron difluoride complexes, 7a-b (Scheme 4). As a point of comparison, and so as to introduce even more electron richness to the ligands at boron centers, the related amino-substituted $\beta$-diketiminato boron fluoride complex, 8, was similarly prepared using $\left[\mathrm{PhC}\left\{\mathrm{C}\left(\mathrm{NEt}_{2}\right) \mathrm{N}(\mathrm{Mes})\right\}_{2}\right] \mathrm{Li}^{3}$ as the transfer reagent.

The spectroscopic data for $\mathbf{7 a - b}$ and $\mathbf{8}$ are consistent with their solid state structures (see below) and all exhibit triplet resonances $\left(J_{\mathrm{BF}}=27-30 \mathrm{~Hz}\right)$ in their ${ }^{11} \mathrm{~B}$ NMR spectra at fields reminiscent of four-coordinate boron centers $(\delta 0.4-1.5 \mathrm{ppm})$. Corresponding quartet resonances were observed in their ${ }^{19} \mathrm{~F}$ NMR spectra. Compounds $7 \mathbf{a}-\mathbf{b}$ have very similar crystal structures, and are structurally comparable with other triazapentadienyl complexes of the $\mathrm{BF}_{2}$ fragment, ${ }^{12 \mathrm{~b}}$ so only the molecular structure of $\mathbf{7 a}$, and that of the structurally distinct $\mathbf{8}$, are shown in Figure 5 (see Supplementary Material for crystallographic details of $\mathbf{7 b}$ ). Both $\mathbf{7 a}$ and $\mathbf{8}$ are monomeric in the solid state, though they differ in that the $\mathrm{C}_{2} \mathrm{~N}_{3} \mathrm{~B}$ heterocycle of $7 \mathbf{a}$ is flat with what appears to be fully delocalized $\mathrm{C}-\mathrm{N}$ bonds, while the boron center of 8 sits significantly above the $\mathrm{C}_{3} \mathrm{~N}_{2}$ backbone fragment (by $0.64 \AA$ ), generating a dihedral angle of $c a .43^{\circ}$ between the NBN and NCCN least squares planes. This ring puckering also forces the mesityl ipso-carbon below the heterocycle, though this apparently does not significantly reduce the degree of electronic delocalization over the $\mathrm{C}_{3} \mathrm{~N}_{2}$, as determined by the bond lengths within that fragment. The distortion from planarity that is observed for the six-membered heterocycle of $\mathbf{8}$ is akin to that reported for other amino-substituted $\beta$-diketiminato complexes of main group elements. ${ }^{3}$ Also, as in those systems, the steric properties of $\mathbf{8}$ do not allow for planarization, and hence significant conjugation, of its backbone $\mathrm{NEt}_{2}$ groups with the $\mathrm{C}_{3} \mathrm{~N}_{2}$ ligand backbone. This must mitigate 
against the enhanced electron richness at the coordinating $\mathrm{N}$-centers that would come with electronic delocalization over the whole planar $\mathrm{N}_{4} \mathrm{C}_{3}$ ligand framework.

(a)
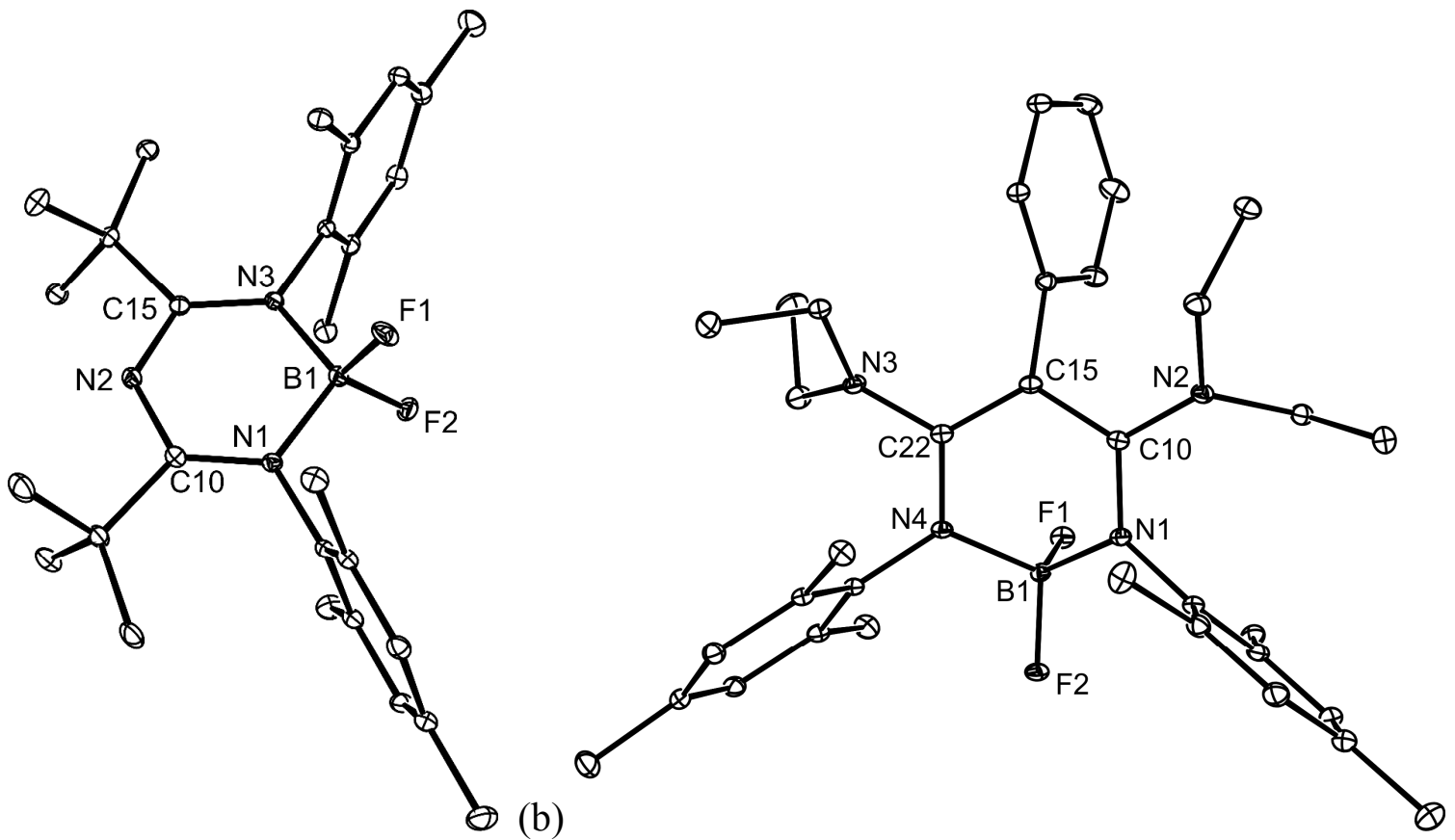

Fig. 5. Thermal ellipsoid plot (25\% probability surface) of the molecular structures of (a) $\left({ }^{\mathrm{Mes}} \mathrm{NNN}\right) \mathrm{BF}_{2} 7 \mathbf{a}$, and (b) $\left\{\mathrm{PhC}\left[\mathrm{C}\left(\mathrm{NEt}_{2}\right) \mathrm{N}(\mathrm{Mes})\right]_{2}\right\} \mathrm{BF}_{2}$ 8. Hydrogen atoms are omitted. Selected bond lengths $(\AA)$ and angles $\left({ }^{\circ}\right)$ for $7 \mathbf{a}: \mathrm{F}(1)-\mathrm{B}(1)$ 1.388(2), $\mathrm{F}(2)-\mathrm{B}(1)$ 1.384(2), $\mathrm{N}(3)-\mathrm{C}(15)$ 1.3286(19)， $\mathrm{N}(3)-\mathrm{B}(1) \quad 1.558(2) ， \mathrm{~N}(1)-\mathrm{C}(10) \quad 1.3277(19) ， \mathrm{~N}(1)-\mathrm{B}(1) \quad 1.559(2) ， \mathrm{~N}(2)-\mathrm{C}(10)$ 1.334(2), N(2)-C(15) 1.335(2), $\mathrm{F}(2)-\mathrm{B}(1)-\mathrm{F}(1)$ 108.61(13), N(3)-B(1)-N(1) 108.18(12); for 8: $\mathrm{F}(1)-\mathrm{B}(1) 1.400(2), \mathrm{B}(1)-\mathrm{F}(2)$ 1.393(2)，N(1)-B(1) 1.556(3)，B(1)-N(4) 1.550(2)，N(1)-C(10) 1.351(2), N(4)-C(22) 1.353(2), $\mathrm{C}(10)-\mathrm{C}(15)$ 1.429(3), $\mathrm{C}(15)-\mathrm{C}(22)$ 1.416(3), $\mathrm{F}(2)-\mathrm{B}(1)-\mathrm{F}(1)$ $110.86(15), \mathrm{N}(4)-\mathrm{B}(1)-\mathrm{N}(1) 107.53(14)$. 
A number of attempts were made to reduce $7 \mathbf{a}-\mathbf{b}$ and $\mathbf{8}$ to give six-membered boron(I) heterocycles, using alkali metals, $\mathrm{KC}_{8}$, sodium naphthalenide, $\mathrm{Mg}$ metal or magnesium(I) dimers as the reducing agent. These attempts led either to intractable mixtures of many products, or no reaction occurring. The one exception here were reductions of toluene or benzene solutions of $\mathbf{7 b}$ with $\mathrm{KC}_{8}$ or a sodium mirror, which gave rise to deep purple solutions. The purple compound in these solutions could not be crystallized, and in solution it appears to be NMR silent. It is also not stable in solution, decomposing over several hours to give a yellow-brown solution which exhibits numerous peaks in its ${ }^{1} \mathrm{H}$ NMR spectrum. We propose that the purple product is a radical species, $\left[\left({ }^{\mathrm{Dep}} \mathrm{NNN}\right) \mathrm{BF}\right] \cdot$ 9. This is closely related to the $\beta$-diketiminato based radical system, $\left[\left\{\mathrm{HC}\left(\mathrm{Bu}^{t} \mathrm{CNDip}\right)_{2}\right\} \mathrm{BF}\right]$; which was formed by reduction of $\left\{\mathrm{HC}\left(\mathrm{Bu}^{t} \mathrm{CNDip}\right)_{2}\right\} \mathrm{BF}_{2}$ with $\mathrm{KC}_{8} .^{19}$ Unfortunately, all attempts to use radical traps, e.g. TEMPO, to isolate a diamagnetic derivative of the purple compound met with failure.

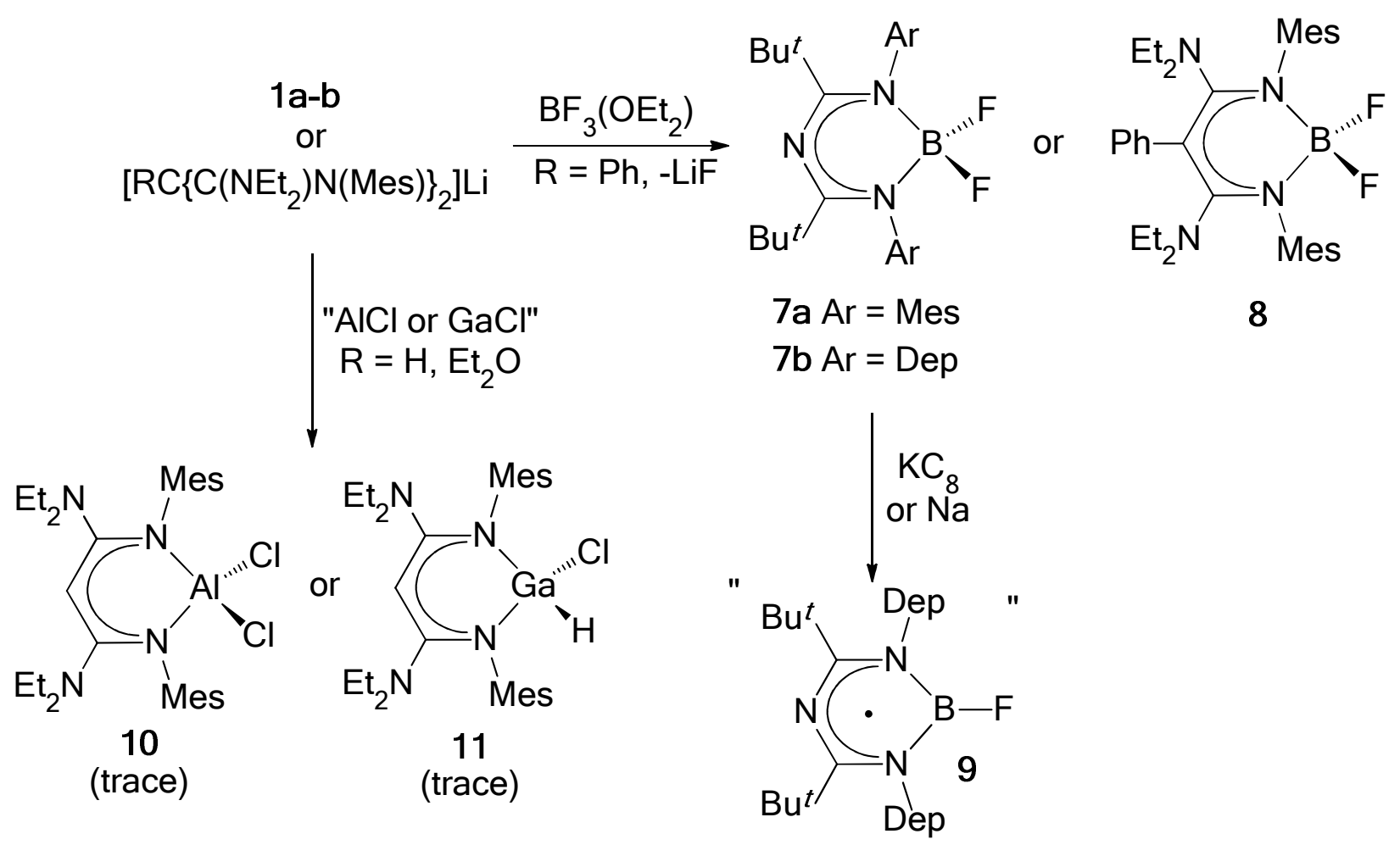

Scheme 4. Synthesis of compounds 7a-b - 11. 
As mentioned above, $\beta$-diketiminates have been successfully utilized to prepare sixmembered metal(I) heterocycles incorporating all of the group 13 metals. Considering this, we explored the ability of triazapentadienyl and amino-substituted $\beta$-diketiminato ligands to do the same. Accordingly, the lithium salts, $\mathbf{1 b}$ and $\left[\mathrm{HC}\left\{\mathrm{C}\left(\mathrm{NEt}_{2}\right) \mathrm{N}(\mathrm{Mes})\right\}_{2}\right] \mathrm{Li}$, were reacted with toluene/diethyl ether suspensions of either $\mathrm{TlCl}$ or $\mathrm{InCl}$, though in all cases unidentified mixtures of soluble products were formed, in addition to the deposition of the group 13 metal. As we have access to "metastable" toluene/THF solutions of $\mathrm{Al}^{\mathrm{I}} \mathrm{Cl}$ and $\mathrm{Ga}^{\mathrm{I}} \mathrm{Cl}^{5}$, these were subsequently reacted with one equivalent of either $\mathbf{1 b}$ or $\left[\mathrm{HC}\left\{\mathrm{C}\left(\mathrm{NEt}_{2}\right) \mathrm{N}(\mathrm{Mes})\right\}_{2}\right] \mathrm{Li}$. While no metal(I) complex was isolated from any reaction, a small number of crystals of the aluminum(III) chloride, 10, and gallium(III) chloride/hydride, 11, were recovered from reactions involving $\left[\mathrm{HC}\left\{\mathrm{C}\left(\mathrm{NEt}_{2}\right) \mathrm{N}(\mathrm{Mes})\right\}_{2}\right] \mathrm{Li}$ (Scheme 4). Given the low yield of these complexes, limited spectroscopic data could be obtained for them. However, both were crystallographically characterized and found to be monomers containing puckered six membered $\mathrm{C}_{3} \mathrm{~N}_{2} \mathrm{M}$ heterocycles with geometries similar to that of $\mathbf{8}$, and amino-substituted $\beta$-diketiminato aluminium alkyl complexes previously reported by us. ${ }^{3}$ As a result, the molecular structures of the compounds have been included in the Supporting Material.

It cannot be sure what the mechanisms of formation of the low yielding compounds, $\mathbf{1 0}$ and 11, are, but the former may result from reaction of $\left[\mathrm{HC}\left\{\mathrm{C}\left(\mathrm{NEt}_{2}\right) \mathrm{N}(\mathrm{Mes})\right\}_{2}\right] \mathrm{Li}$ with traces of $\mathrm{AlCl}_{3}$ that arise from slow disproportionation of $\mathrm{Al}^{\mathrm{I}} \mathrm{Cl}$ that occurred upon storing its toluene/THF solutions at $-80{ }^{\circ} \mathrm{C}$ over approximately 1 year. In contrast, a plausible mechanism of formation for 11 could involve oxidative addition of the N-H bond of traces of the protonated ligand, $\mathrm{HC}\left\{\mathrm{C}\left(\mathrm{NEt}_{2}\right) \mathrm{NH}(\mathrm{Mes})\right\}\left\{\mathrm{C}\left(\mathrm{NEt}_{2}\right) \mathrm{N}(\mathrm{Mes})\right\}$ (present in solutions of $\left.\left[\mathrm{HC}\left\{\mathrm{C}\left(\mathrm{NEt}_{2}\right) \mathrm{N}(\mathrm{Mes})\right\}_{2}\right] \mathrm{Li}\right)$, to 
the gallium(I) centre of $\mathrm{Ga}^{\mathrm{I}} \mathrm{Cl} \cdot{ }^{17}$ Whatever the case, all subsequent attempts to rationally prepare 10 and 11, by reaction of $\left[\mathrm{HC}\left\{\mathrm{C}\left(\mathrm{NEt}_{2}\right) \mathrm{N}(\mathrm{Mes})\right\}_{2}\right] \mathrm{Li}$ with $\mathrm{AlCl}_{3}$, and treatment of $\mathrm{Ga}^{\mathrm{I}} \mathrm{Cl}$ solutions with $\mathrm{HC}\left\{\mathrm{C}\left(\mathrm{NEt}_{2}\right) \mathrm{NH}(\mathrm{Mes})\right\}\left\{\mathrm{C}\left(\mathrm{NEt}_{2}\right) \mathrm{N}(\mathrm{Mes})\right\}$, led to product mixtures from which no pure compound could be isolated.

\section{Conclusions}

In summary, three extremely bulky 1,3,5-triazapentadienes have been prepared and structurally characterized. These are readily deprotonated, yielding a series of lithium and potassium triazapentadienyl complexes, which can be used to transfer the ligand to other metal centers. Moreover, deprotonation of the triazapentadienes with MeMgI has given three triazapentadienyl magnesium iodide complexes, the crystal structures of which reveal them to be monomeric. In contrast, a related triazapentadienyl calcium iodide complex has been prepared and shown to be an iodide bridged dimer in the solid state. Attempts to reduce the magnesium iodide complexes led to product mixtures, the main components of which are homoleptic bis(triazapentadienyl) magnesium complexes. From one reaction a very low yield of a triazapentadienyl coordinated magnesium(I) dimer was isolated and structurally characterized. In related chemistry, two triazapentadienyl boron difluoride compounds have been synthesized. Reduction of one of these with alkali metal reagents has given a deeply colored, likely radical, compound of indeterminate formulation. For sake of comparison, attempts have been made to prepare a series of amino-substituted $\beta$-diketiminato group 13 element(I) heterocycles. Although this was not achieved, several group 13 element(III) halide complexes incorporating this ligand class have been characterized, either as precursor complexes, or as reduction by-products. We 
continue to explore the development of extremely bulky anionic $N$-donor ligands, and their application to the stabilization of low oxidation state metal complexes.

Acknowledgement. This research was supported by the Australian Research Council and the US Air Force Asian Office of Aerospace Research and Development (grant FA2386-14-1-4043 to CJ). Part of this research was undertaken on the MX1 beamline at the Australian Synchrotron, Victoria, Australia.

Supplementary Material. Supplementary data are available with the article through the journal Web site at http://nrcresearchpress.com/doi/suppl/10.1139/cjc-xxxxx. CCDC 150509-1570524 contain the crystallograhic data in CIF format for this article. These data can be obtained, free of charge, via http://www.ccdc.cam.ac.uk/products/csd/ request (or from the Cambridge Crystallographic Data Centre, 12 Union Road, Cambridge CB2 1E2, UK; fax: +44 1223 33603; e-mail: deposit@ccdc.cam.ac.uk). 


\section{References.}

(1) Selected reviews (a) Review: Fischer, R. C.; Power, P. P. Chem. Rev. 2010, 110, 3877. doi:10.1021/cr100133q; (b) Asay, M.; Jones, C.; Driess, M. Chem. Rev. 2011, 111, 354. doi: 10.1021/cr100216y; (c) Power, P. P. Acc. Chem. Res. 2011, 44, 627. doi:10.1021/ar2000875; (d) Yao, S.; Xiong, Y.; Driess, M. Organometallics 2011, 30, 1748. doi:10.1021/om200017h (e) Power, P. P. Nature 2010, 463, 171. doi:10.1038/nature08634.

(2) Selected reviews (a) Bourget-Merle, L.; Lappert, M. F.; Severn, J. R. Chem. Rev. 2002, 102, 3031. doi:10.1021/cr010424r; (b) Tsai, Y.-C. Coord. Chem. Rev. 2012, 256, 722. doi.org/10.1016/j.ccr.2011.12.012; (c) Baker, R. J.; Jones, C. Coord. Chem. Rev. 2005, 249, 1857. doi:10.1016/j.ccr.2004.12.016.

(3) Do, D. C. H.; Keyser, A.; Protchenko, A. V.; Maitland, B.; Pernik, I.; Niu, H.; Kolychev, E. L.; Rit, A.; Vidovic, D.; Stasch, A.; Jones, C.; Aldridge, S. Chem. Eur. J. 2017, 23, 5830. doi:10.1002/chem.201700757.

(4) Selected recent examples (a) Kirubakaran, B.; Beesam, R.; Nareddula, D. R. Appl. Organomet. Chem. 2017, e3833. doi.org/10.1002/aoc.3833; (b) Bakthavachalam, K.; Reddy, N. D. Organometallics 2013, 32, 3174. doi:org/10.1021/om3011432; (c) Kulkarni, N. V.; Das, A.; Ridlen, S. G.; Maxfield, E.; Adiraju, V. A. K.; Yousufuddin, M.; Dias, H.V.R. Dalton Trans. 2016, 45, 4896. doi:10.1039/C6DT00257A; (d) Dias, H.V.R.; Singh, S.; Cundari, T. R. Angew. Chem. Int. Ed. 2005, 44, 4907. doi:10.1002/anie.200500401.

(5) Dange, D.; Li, J.; Schenk, C.; Schnöckel, H.; Jones, C. Inorg. Chem. 2012, 51, 13050. doi:10.1021/ic3022613. 
(6) Budzelaar, P. H. M.; van Oort, A. B.; Orpen, A. G. Eur. J. Inorg. Chem. 1998, 1485. doi:10.1002/(SICI)1099-0682(199810)1998:10<1485::AID-EJIC1485>3.0.CO;2-F.

(7) van Dijk, T.; Bakker, M. S.; Holtrop, F.; Nieger, M.; Slootweg, J. C.; Lammertsma, K. Org. Lett. 2015, 17, 1461. doi:10.1021/acs.orglett.5b00339.

(8) Kalden, D.; Oberheide, A.; Loh, C.; Görls, H.; Kriek, S.; Westerhausen, M. Chem. Eur. J. 2016, 22, 10944. doi:10.1002/chem.201602074.

(9) McPhillips, T. M.; McPhillips, S. E.; Chiu, H. J.; Cohen, A. E.; Deacon, A. M.; Ellis, P. J.; Garman, E.; Gonzalez, A.; Sauter, N. K.; Phizackerley, R. P.; Soltis, S. M.; Kuhn, P. J. Synchrotron Rad. 2002, 9, 401. doi:10.1107/S0909049502015170.

(10) Kabsch, W. J. Appl. Cryst. 1993, 26, 795. doi:10.1107/S0021889893005588.

(11) Sheldrick, G. M. SHELX-97, University of Göttingen, 1997.

(12) Selected examples (a) Flores, J. A.; Kobayashi, Y.; Dias, H. V. R. Dalton Trans. 2011, 40, 10351. doi:10.1039/c1dt10199d; (b) Hager, I.; Frohlich, R.; Wurthwein, E. -U. Eur. J. Inorg. Chem. 2009, 2415. doi:10.1002/ejic.200900042; (c) Dias, H. V. R.; Singh, S. Inorg. Chem. 2004, 43, 5786. doi:10.1021/ic049229w; (d) Dias, H. V. R.; Singh, S. Dalton Trans. 2006, 1995. doi:10.1039/b518464a.

(13) (a) Stender, M.; Wright, R. J.; Eichler, B. E.; Prust, J.; Olmstead, M. M.; Roesky, H. W.; Power, P. P. J. Chem. Soc., Dalton Trans. 2001, 3465. doi:10.1039/b103149j; (b) Takeda, N.; Hamaki, H.; Tokitoh, N. Chem. Lett. 2004, 33, 134. doi:10.1246/cl.2004.134.

(14) (a) Jones, C. Nat. Rev. Chem. 2017, 1, 0059. doi:10.1038/s41570-017-0059; (b) Jones, C.; Stasch, A. Top. Organomet. Chem. 2013, 45, 73. doi:10.1007/978-3-642-36270-5_3; (c) Stasch, A.; Jones, C. Dalton Trans. 2011, 40, 5659. doi:10.1039/c0dt01831g.

(15) See for example (a) Bonyhady, S. J.; Jones, C.; Nembenna, S.; Stasch, A.; Edwards, A. J.; McIntyre, G. J. Chem. Eur. J. 2010, 16, 938. doi:10.1002/chem.200902425; (b) 
Lalrempuia, R.; Kefalidis, C. E.; Bonyhady, S. J.; Schwarze, B.; Maron, L.; Stasch, A.; Jones, C. J. Am. Chem. Soc. 2015, 137, 8944. doi:10.1021/jacs.5b06439.

(16) Emsley, J. The Elements, 2nd ed., Clarendon, Oxford, 1995.

(17) Jones, C.; Stasch, A. in The Group 13 Metals Aluminium, Gallium, Indium and Thallium. Chemical Patterns and Peculiarities, Chapter 5, Downs, A. J; Aldridge, S. (eds.), WileyBlackwell, Chichester, 2011, pp 285-341. isbn:978-00470-68191-6.

(18) (a) Reiher, M.; Sundermann, A. Eur. J. Inorg. Chem. 2002, 1854. doi:10.1002/10990682(200207)2002:7<1854::AID-EJIC1854>3.0.CO;2-1 ; (b) Chen, C.-H.; Tsai, M.-L.; Su, M.D. Organometallics 2006, 25, 2766. doi:10.1021/om0600011.

(19) Aramaki,Y.; Omiya, H.; Yamashita, M.; Nakabayashi, K.; Ohkoshi, S.-I.; Nozaki, K. J. Am. Chem. Soc. 2012, 134, 19989. doi:10.1021/ja3094372. 


\title{
Synthesis and Attempted Reductions of Bulky 1,3,5-Triazapentadienyl Groups 2 and 13 Halide Complexes
}

\author{
Indrek Pernik, ${ }^{\mathrm{a}}$ Brant J. Maitland, ${ }^{\mathrm{a}}$ Andreas Stasch*,a,b and Cameron Jones* ${ }^{* \mathrm{a}}$ \\ ${ }^{a}$ School of Chemistry, Monash University, P.O. Box 23, Melbourne, Victoria, 3800, Australia \\ Email: cameron.jones@monash.edu,Ph:+61-3-9902-0391, Fax:+61-3-9905-4597 \\ Web: www.monash.edu/science/research-groups/chemistry/jonesgroup \\ ${ }^{b}$ Current address: EaStCHEM School of Chemistry, University of St Andrews, North Haugh, \\ KY16 9ST, St Andrews, United Kingdom
}

\section{TABLE OF CONTENTS ENTRY ONLY}

Several extremely bulky 1,3,5-triazapentadienes have been prepared and utilized in the preparation of a range of monomeric, groups 2 and 13 element halide complexes. Attempts to prepare low oxidation state main group complexes by reduction of these precursor compounds were largely unsuccessful. However, a low yield of a new magnesium(I) dimer (see picture), stabilized by 1,3,5-triazapentadienyl ligands, was obtained and the compound crystallographically characterized.

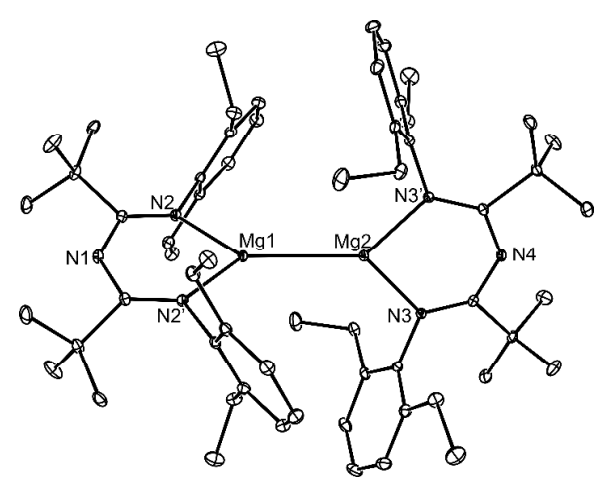

